\title{
2011s-21
}

\section{Identification-robust estimation and testing of the zero-beta CAPM}

\author{
Marie-Claude Beaulieu, Jean-Marie Dufour, Lynda Khalaf
}

\begin{tabular}{c}
\hline Série Scientifique \\
Scientific Series
\end{tabular}

Montréal

Février 2011

(C) 2011 Marie-Claude Beaulieu, Jean-Marie Dufour, Lynda Khalaf. Tous droits réservés. All rights reserved. Reproduction partielle permise avec citation du document source, incluant la notice (C).

Short sections may be quoted without explicit permission, if full credit, including (C) notice, is given to the source.
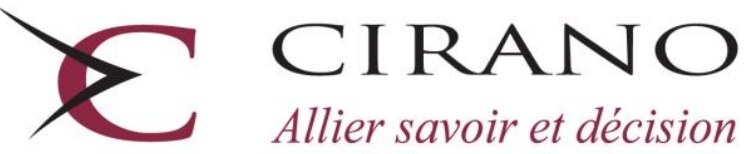

Allier savoir et décision

Centre interuniversitaire de recherche en analyse des organisations 


\section{CIRANO}

Le CIRANO est un organisme sans but lucratif constitué en vertu de la Loi des compagnies du Québec. Le financement de son infrastructure et de ses activités de recherche provient des cotisations de ses organisations-membres, d'une subvention d'infrastructure du Ministère du Développement économique et régional et de la Recherche, de même que des subventions et mandats obtenus par ses équipes de recherche.

CIRANO is a private non-profit organization incorporated under the Québec Companies Act. Its infrastructure and research activities are funded through fees paid by member organizations, an infrastructure grant from the Ministère du Développement économique et régional et de la Recherche, and grants and research mandates obtained by its research teams.

\section{Les partenaires du CIRANO}

Partenaire majeur

Ministère du Développement économique, de l'Innovation et de l'Exportation

\section{Partenaires corporatifs}

Banque de développement du Canada

Banque du Canada

Banque Laurentienne du Canada

Banque Nationale du Canada

Banque Royale du Canada

Banque Scotia

Bell Canada

BMO Groupe financier

Caisse de dépôt et placement du Québec

Fédération des caisses Desjardins du Québec

Financière Sun Life, Québec

Gaz Métro

Hydro-Québec

Industrie Canada

Investissements PSP

Ministère des Finances du Québec

Power Corporation du Canada

Raymond Chabot Grant Thornton

Rio Tinto

State Street Global Advisors

Transat A.T.

Ville de Montréal

\section{Partenaires universitaires}

École Polytechnique de Montréal

HEC Montréal

McGill University

Université Concordia

Université de Montréal

Université de Sherbrooke

Université du Québec

Université du Québec à Montréal

Université Laval

Le CIRANO collabore avec de nombreux centres et chaires de recherche universitaires dont on peut consulter la liste sur son site web.

Les cahiers de la série scientifique (CS) visent à rendre accessibles des résultats de recherche effectuée au CIRANO afin de susciter échanges et commentaires. Ces cahiers sont écrits dans le style des publications scientifiques. Les idées et les opinions émises sont sous l'unique responsabilité des auteurs et ne représentent pas nécessairement les positions du CIRANO ou de ses partenaires.

This paper presents research carried out at CIRANO and aims at encouraging discussion and comment. The observations and viewpoints expressed are the sole responsibility of the authors. They do not necessarily represent positions of CIRANO or its partners. 


\title{
Identification-robust estimation and testing of the zero-beta CAPM ${ }^{*}$
}

\author{
Marie-Claude Beaulieu ${ }^{\dagger}$, Jean-Marie Dufour ${ }^{+}$, Lynda Khalaf ${ }^{\S}$
}

\begin{abstract}
We propose exact simulation-based procedures for: (i) testing mean-variance efficiency when the zerobeta rate is unknown, and (ii) building confidence intervals for the zero-beta rate. On observing that this parameter may be weakly identified, we propose LR-type statistics as well as heteroskedascity and autocorrelation corrected (HAC) Wald-type procedures, which are robust to weak identification and allow for non-Gaussian distributions including parametric GARCH structures. In particular, we propose confidence sets for the zero-beta rate based on "inverting" exact tests for this parameter; these sets provide a multivariate extension of Fieller's technique for inference on ratios. The exact distribution of LR-type statistics for testing efficiency is studied under both the null and the alternative hypotheses. The relevant nuisance parameter structure is established and finite-sample bound procedures are proposed, which extend and improve available Gaussianspecific bounds. Furthermore, we study the invariance to portfolio repacking property for tests and confidence sets proposed. The statistical properties of available and proposed methods are analyzed via aMonte Carlo study. Empirical results on NYSE returns show that exact confidence sets are very different from the asymptotic ones, and allowing for non-Gaussian distributions affects inference results. Simulation and empirical results suggest that LR-type statistics - with p-values corrected using the Maximized Monte Carlo test method - are generally preferable to their Wald-HAC counterparts from the viewpoints of size control and power.
\end{abstract}

Key words: capital asset pricing model, CAPM; Black, mean-variance efficiency, non-normality, weak identification, Fieller, multivariate linear regression, uniform linear hypothesis, exact test, Monte Carlo test, bootstrap, nuisance parameters, GARCH, portfolio repacking.

JEL codes: C3, C12, C33, C15, G1, G12, G14.

\footnotetext{
* The authors thank two anonymous referees and the Editor, Bernard Salanié, for several useful comments. This work was supported by the William Dow Chair in Political Economy (McGill University), the Chaire RBC en innovations financières (Université Laval), the Canada Research Chair Program, the Bank of Canada (Research Fellowship), a Guggenheim Fellowship, a Konrad-Adenauer Fellowship (Alexander-von-Humboldt Foundation, Germany), the Institut de finance mathématique de Montréal (IFM2), the Canadian Network of Centres of Excellence [program on Mathematics of Information Technology and Complex Systems (MITACS)], the Natural Sciences and Engineering Research Council of Canada, the Social Sciences and Humanities Research Council of Canada, the Fonds de recherche sur la société et la culture and NATECH (Government of Québec).

${ }^{\dagger}$ Chaire RBC en innovations financières, Centre interuniversitaire sur le risque, les politiques économiques et l'emploi (CIRPÉE), Département de finance et assurance, Pavillon Palasis-Prince, local 3620-A, Université Laval, Québec, Canada G1K 7P4. TEL: (418) 656-2131-2926, FAX: (418) 656-2624. email: Marie-Claude.Beaulieu@ fas.ulaval.ca ${ }^{\star}$ William Dow Professor of Economics, McGill University, Centre interuniversitaire de recherche en analyse des organisations (CIRANO), and Centre interuniversitaire de recherche en économie quantitative (CIREQ).Mailing address: Department of Economics, McGill University, Leacock Building, Room 519, 855 Sherbrooke Street West, Montréal, Québec H3A 2T7, Canada. TEL: (1) 514398 8879; FAX: (1) 514398 4938; e-mail: jeanmarie.dufour@mcgill.ca. Webpage: http://www.jeanmariedufour.com

${ }^{\S}$ Groupe de recherche en économie de l'énergie, de l'environnement et des ressources naturelles (GREEN) Université Laval, Centre interuniversitaire de recherche en économie quantitative (CIREQ), and Economics Department, Carleton University. Mailing address: Economics Department, Carleton University, Loeb Building 1125 Colonel By Drive, Ottawa, Ontario, K1S 5B6 Canada. Tel (613) 520-2600-8697; FAX: (613)-520-3906. email: Lynda_Khalaf@carleton.ca.
} 


\section{Introduction}

One of the most important extensions of the Capital Asset Pricing Model (CAPM) consists in allowing for the absence of a risk-free asset. From a theoretical viewpoint, this can be due to restrictions on borrowing [Black (1972)] or an investor's riskless borrowing rate that exceeds the Treasury bill rate [Brennan (1971)]. In this case, portfolio mean-variance efficiency is defined using the expected return in excess of the zero-beta portfolio. The latter is however unobservable which leads to considerable empirical difficulties.

Indeed, there are two basic approaches to estimating and assessing this version of the CAPM (denoted below as BCAPM). The first one uses a "two-pass" approach that may be traced back to Black, Jensen and Scholes (1972) and Fama and MacBeth (1973): betas are first estimated from time series regressions for each security, and then the zero-beta rate is estimated by a cross-sectional regression on these betas. This raises errors-in-variables problems that affect statistical inference in both finite and large samples. ${ }^{1}$ The second approach - which appears in the seminal work of Jensen (1968) - avoids this problem by using as statistical framework a multivariate linear regression (MLR). ${ }^{2}$ In this paper, we focus on the MLR approach and consider two basic problems: (1) testing portfolio efficiency; (2) building a reliable confidence set (CS) for the zero-beta rate.

For clarity, let $R_{i t}, i=1, \ldots, n$, be the returns on $n$ securities in period $t$, and $\tilde{R}_{\mathrm{M} t}$ the return on a market benchmark for $t=1, \ldots, T$, and consider the $n$ equations $(i=1, \ldots, n)$ associated with the time series regressions of $R_{i t}$ on a constant and $\tilde{R}_{\mathrm{M} t}$, where the individualequation disturbances are heteroskedastic and contemporaneously cross-correlated; let $\Sigma=K^{\prime} K$ refer to the error scale (or variance/covariance) matrix. If the intercepts from these $n$ equations (the alphas) are denoted $a_{i}$, and the coefficients on the benchmark regressor (the betas) are denoted $\beta_{i}$, $i=1, \ldots, n$, then the BCAPM equilibrium relations imply the following: there is a scalar $\gamma$, the return on the zero-beta portfolio, such that $a_{i}-\gamma\left(1-\beta_{i}\right)=0, i=1, \ldots, n$. Our aim consists in assessing these constraints (denoted below as $\mathcal{H}_{\mathrm{B}}$ ) as well as estimating $\gamma$.

The above cited literature provides analytical formulae for Gaussian likelihood-ratio (LR) statistics, the maximum likelihood estimator (MLE) of $\gamma$ (denoted below as $\hat{\gamma}$ ), and for a conformable asymptotic variance estimator [denoted below as $\operatorname{Var}(\hat{\gamma})$ ]. It is however difficult to find reliable critical points in this context. While Gibbons (1982) used an asymptotic $\chi^{2}$ critical value for the LR statistic, subsequent authors found this could lead to serious over-rejections, so various finitesample corrections - such as bounds - have been suggested; see Shanken $(1985,1986,1996)$, Stewart (1997), Zhou (1991, 1995), and Velu and Zhou (1999). These corrections depend crucially on normality, which may be inappropriate for financial data [see Fama (1965), Richardson and Smith (1993), Dufour, Khalaf and Beaulieu (2003) and Beaulieu, Dufour and Khalaf (2005, 2007, 2009)]. Furthermore, evidence on the properties of the confidence interval based on $\operatorname{Var}(\hat{\gamma})$ is unavailable. Despite the simplicity of the above framework, discrepancies between asymptotic and finite sample distributions are not surprising. Indeed, three difficulties deserve notice.

(1) Dimensionality: as $n$ increases, the dimension of the scale matrix $\Sigma$ grows rapidly and available

\footnotetext{
${ }^{1}$ See e.g. Litzenberger and Ramaswamy (1979), Banz (1981), Roll (1985), Chen, Roll and Ross (1986), Shanken (1992), Kim (1995), Shanken and Zhou (2007), Lewellen, Nagel and Shanken (2009), Kan, Robotti and Shanken (2008), and Kleibergen (2009).

${ }^{2}$ For other work based on the MLR approach to CAPM analysis, see Gibbons (1982), Jobson and Korkie (1982), Kandel (1984, 1986), Amsler and Schmidt (1985), Shanken (1985, 1986, 1996), Kandel and Stambaugh (1989), Zhou (1991), Shanken (1992), Fama and French (1993), Chou (2000), Fama and French (2004) and Perold (2004).
} 
degrees-of-freedom decrease conformably. ${ }^{3}$ Even in linear or standard setups where the relevant asymptotic distributions may be free of $\Sigma$, this matrix can still affect the distributions in finite samples. Furthermore, positive definite estimates of $\Sigma$ require a large $T$ relative to $n$, so portfolios rather than securities are often used in practice.

(2) Portfolio repacking [see Kandel and Stambaugh (1989)]: to preserve meaningful pricing relations when portfolios are used, transformations of the return vector $R_{t}=\left(R_{1 t}, \ldots, R_{n t}\right)^{\prime}$ into $R_{t}^{*}=A R_{t}$ where $A$ is an $n \times n$ invertible matrix such that $A \iota_{n}=\iota_{n}$ and $\iota_{n}$ is an $n$-dimensional vector of ones, should ideally not affect inference.

(3) Identification: as $\beta_{i} \rightarrow 1, \gamma$ becomes weakly identified. Weak identification strongly affects the distributions of estimators and test statistics, leading to asymptotic failures. ${ }^{4}$ This should not be taken lightly, for although reported betas [see e.g. Fama and MacBeth (1973)] are often close to one, in view of properties (1) and (2), one may not assume irregularities away even when estimated betas are not close to one. Indeed, in the regression of $R_{t}^{*}$ [from (2)] on a constant and $\tilde{R}_{\mathrm{M} t}$, with intercepts $a_{i}^{*}$ and slopes $\beta_{i}^{*}, a_{i}-\gamma\left(1-\beta_{i}\right)=0, i=1, \ldots, n \Leftrightarrow a_{i}^{*}-\gamma\left(1-\beta_{i}^{*}\right)=0, i=1, \ldots, n$ for any $\gamma$ and $A$. Portfolio repacking alters betas along with scale yet preserves the definition of $\gamma$, leading to identification problems as $\beta_{i}^{*} \rightarrow 1$. So the betas and scale parameters play a role in identifying $\gamma$.

Our aim in this paper consists in providing inference methods that are robust to dimensionality and identification problems, whose outcomes are invariant to portfolio repacking. We first consider the problem of estimating $\gamma$. We show by simulation that available procedures provide poor coverage. So we propose exact CSs based on "inverting" exact tests for specific values of $\gamma$, i.e. the set of values not rejected by these tests. This method is a generalization of the classical procedure proposed by Fieller (1954) to estimate parameter ratios. ${ }^{5}$

To introduce the Fieller-type method in its simplest form with reference to the problem at hand, suppose (for illustration sake) that we aim at estimating $\gamma$ from the univariate regression of the return of the $i$-th security $\left(R_{i t}\right)$ on a constant and $\tilde{R}_{\mathrm{M} t}$, so that $\gamma=-a_{i} / \delta_{i}$ where $\delta_{i}=\left(\beta_{i}-1\right)$. Let $\hat{a}_{i}$ and $\hat{\delta}_{i}$ denote the OLS estimates from this regression, with estimated variances and covariance $\operatorname{Var}\left(\hat{a}_{i}\right), \operatorname{Var}\left(\hat{\delta}_{i}\right)$ and $\operatorname{Cov}\left(\hat{a}_{i}, \hat{\delta}_{i}\right)$. For each possible value $\gamma_{0}$ of the ratio, consider the t-statistic $\mathrm{t}_{i}\left(\gamma_{0}\right)=\left(\hat{a}_{i}+\gamma_{0} \hat{\delta}_{i}\right) /\left[\operatorname{Var}\left(\hat{a}_{i}\right)+\delta_{0}^{2} \operatorname{Var}\left(\hat{\delta}_{i}\right)+2 \delta_{0} \operatorname{Cov}\left(\hat{a}_{i}, \hat{\delta}_{i}\right)\right]^{1 / 2}$ for testing $\mathcal{H}_{i}\left(\gamma_{0}\right): a_{i}+\gamma_{0} \delta_{i}=0$. Then, we obtain a CS with level $1-\alpha$ for $\gamma$ by finding the set of $\gamma_{0}$ values which are not rejected at level $\alpha$ using $\mathrm{t}_{i}\left(\gamma_{0}\right)$ and a standard normal two-tailed critical value $\mathrm{z}_{\alpha / 2}$. This means that we collect all $\gamma_{0}$ values such that $\left|t_{i}\left(\gamma_{0}\right)\right| \leq z_{\alpha / 2}$ or alternatively such that $\left(\hat{a}_{i}+\gamma_{0} \hat{\delta}_{i}\right)^{2} \leq z_{\alpha / 2}^{2}\left(\operatorname{Var}\left(\hat{a}_{i}\right)+\right.$ $\left.\delta_{0}^{2} \operatorname{Var}\left(\hat{\delta}_{i}\right)+2 \delta_{0} \operatorname{Cov}\left(\hat{a}_{i}, \hat{\delta}_{i}\right)\right)$, leading to a second degree inequality in $\gamma_{0}$. The resulting CS has level $1-\alpha$ irrespective whether $\delta_{i}$ is zero or not. In this paper, we generalize this method to account for the multivariate definition of $\gamma$ as described above, in Gaussian and non-Gaussian settings, as well as allowing for conditional heteroskedasticity. Empirically, we focus on multivariate Student- $\boldsymbol{t}$ and normal mixture distributions, as well as Gaussian GARCH.

\footnotetext{
${ }^{3}$ See Shanken (1996), Campbell, Lo and MacKinlay (1997), Dufour and Khalaf (2002), Beaulieu, Dufour and Khalaf (2005, 2007, 2009), Sentana (2009), and the references therein.

${ }^{4}$ See, e.g. Dufour (1997, 2003), Staiger and Stock (1997), Wang and Zivot (1998), Zivot, Startz and Nelson (1998), Dufour and Jasiak (2001), Kleibergen (2002, 2005, 2009), Stock, Wright and Yogo (2002), Moreira (2003), Dufour and Taamouti $(2005,2007)$ and Andrews, Moreira and Stock (2006).

${ }^{5}$ For the ratio of the means of two normal variables with equal variances, Fieller gave a solution that avoids nonregularities arising from a close-to-zero denominator. Extensions to univariate regressions or to several ratios with equal denominators can be found in Zerbe (1978), Dufour (1997), Bolduc, Khalaf and Yelou (2008).
} 
To do so, we consider two statistics [denoted $L R\left(\gamma_{0}\right)$ and $\mathcal{J}\left(\gamma_{0}\right)$ below] for testing $\mathcal{H}\left(\gamma_{0}\right)$ : $a_{i}+\gamma_{0} \delta_{i}=0, i=1, \ldots, n . \operatorname{LR}\left(\gamma_{0}\right)$ is the likelihood ratio (LR) derived from the Gaussian error model, while $\mathcal{J}\left(\gamma_{0}\right)$ is a heteroskedascity and autocorrelation corrected (HAC) multivariate Wald statistic [see e.g. MacKinlay and Richardson (1991), Ravikumar, Ray and Savin (2000), and Ray and Savin (2008)]. Using any one of these tests, we can build confidence sets by finding the values of $\gamma_{0}$ which are not rejected at level $\alpha$. This requires a distributional theory for the test statistics. While an $F$-based cut-off point is available for $\operatorname{LR}\left(\gamma_{0}\right)$ in the i.i.d. Gaussian case [see Beaulieu, Dufour and Khalaf (2007) and Gibbons, Ross and Shanken (1989)], we show in a simulation study that usual asymptotic critical points perform poorly especially for $\mathcal{J}\left(\gamma_{0}\right)$. To deal with such difficulties, we apply the maximized Monte Carlo (MMC) test procedure [Dufour (2006)] to obtain finite-sample p-values for $\operatorname{LR}\left(\gamma_{0}\right)$ and $\mathcal{J}\left(\gamma_{0}\right)$ in models with non-Gaussian and/or non-i.i.d. errors, as follows: a (simulated) $p$-value function conditional on relevant nuisance parameters is numerically maximized (with respect to these parameters), and the test is significant at level $\alpha$ if the largest $p$-value is not larger than $\alpha .^{6}$

To implement this approach efficiently, it is important to characterize the nuisance parameters in the null distributions of the test statistics. We show that the null distribution of $L R\left(\gamma_{0}\right)$ does not depend on $B$ and $\Sigma$, so the only nuisance parameters are: the degrees-of-freedom for the Student- $t$ distribution, the mixing probability and scale-ratio parameters for normal mixtures, or the GARCH parameters. The parametric bootstrap relates to the MMC method, in the sense that the maximization step is replaced by a unique $p$-value estimation, based on a consistent nuisance parameter estimate. For the GARCH case, such estimates may be unreliable in high-dimensional models; we show that the MMC method avoids this problem, with minimal power costs.

Because an $F$-based exact cut-off is available for the Gaussian case, we show that the CS which inverts $L R\left(\gamma_{0}\right)$ can be obtained by solving a quadratic inequation. For non-i.i.d. or non-Gaussian distributions, we implement a numerical search running the MMC method for each choice for $\gamma_{0}$. Furthermore, we show that all proposed CSs provide relevant information on whether efficiency is supported by the data, a property not shared by standard confidence intervals. Indeed, our CSs may turn out to be empty, which occurs when all possible values of $\gamma$ are rejected.

We next consider testing efficiency in the BCAPM context. We study LR and Wald-HAC criteria based on minimizing (over $\left.\gamma_{0}\right)$ the above defined $\operatorname{LR}\left(\gamma_{0}\right)$ and $\mathcal{J}\left(\gamma_{0}\right)$ statistics. We show that the exact distribution of $\min _{\gamma_{0}}\left\{L R\left(\gamma_{0}\right)\right\}$ depends on a reduced number of nuisance parameters which are functions of both $B$ and $\Sigma$. We also generalize Shanken's (1986) exact bound test beyond the Gaussian model, and propose a tighter bound, which involves a numerical search for the tightest cut-off point, based on the MMC method. The MMC based bound is also extended to the $\min _{\gamma_{0}}\left\{\mathcal{J}\left(\gamma_{0}\right)\right\}$ case. This approach, in conjunction with the above defined CS based on $\mathcal{J}\left(\gamma_{0}\right)$, provides an interesting alternative to available GMM estimation methods [including the case recently analyzed by Shanken and Zhou (2007)].

We conduct a simulation study to document the properties of our proposed procedures relative to available ones. In particular, we contrast problems arising from small samples with those caused by fundamentally flawed asymptotics. We next examine efficiency of the market portfolio for monthly returns on New York Stock Exchange (NYSE) portfolios, built from the University of Chicago Center for Research in Security Prices (CRSP) 1926-1995 data base. We find more support for

\footnotetext{
${ }^{6}$ This procedure is based on the following fundamental property: when the distribution of a test statistic depends on nuisance parameters, the desired level $\alpha$ is achieved by comparing the largest $p$-value (over all nuisance parameters consistent with the null hypothesis) with $\alpha$.
} 
efficiency under the non-normal or non-i.i.d. hypothesis. Exact CSs for $\gamma$ considerably differ from asymptotic ones, and Wald-HAC based CSs are much wider than the GARCH corrected LR-based ones.

The paper is organized as follows. Section 2 sets the framework and discusses identification of $\gamma$. In Section 3, we propose finite-sample tests for specific values of $\gamma$, and the corresponding exact CS are derived in Section 4. The exact distribution of the LR efficiency test statistic is established in Section 5, and bound procedures are proposed in Section 6. The simulation study is reported in Section 7. Our empirical analysis is presented in Section 8. We conclude in Section 9.

\section{Framework and identification of $\gamma$}

Let $R_{i t}, i=1, \ldots, n$, be the returns on $n$ securities in period $t$, and $\tilde{R}_{\mathrm{M} t}$ the return on a market benchmark $(t=1, \ldots, T)$. Our analysis of the BCAPM model is based on the following standard MLR setup [Gibbons (1982), Shanken (1986), MacKinlay (1987)]:

$$
R_{i t}-\tilde{R}_{\mathrm{M} t}=a_{i}+\left(\beta_{i}-1\right) \tilde{R}_{\mathrm{M} t}+u_{i t}, \quad i=1, \ldots, n, t=1, \ldots, T,
$$

where $u_{i t}$ are random disturbances. The testable implication of the BCAPM on (2.1) is the following one: there is a scalar $\gamma$, the return on the zero-beta portfolio, such that

$$
\mathcal{H}_{\mathrm{B}}: a_{i}+\gamma \delta_{i}=0, \quad \delta_{i}=\beta_{i}-1, \quad i=1, \ldots, n, \quad \text { for some } \gamma \in \Gamma,
$$

where $\Gamma$ is some set of "admissible" values for $\gamma$. Since $\gamma$ is unknown, $\mathcal{H}_{\mathrm{B}}$ is nonlinear. The latter can be viewed as the union of more restrictive linear hypotheses of the form

$$
\mathcal{H}\left(\gamma_{0}\right): a_{i}+\gamma_{0} \delta_{i}=0, i=1, \ldots, n,
$$

where $\gamma_{0}$ is specified. This observation will underlie our exact inference approach.

The above model is a special case of the following MLR:

$$
Y=X B+U
$$

where $Y=\left[Y_{1}, \ldots, Y_{n}\right]$ is $T \times n, X$ is $T \times k$ of rank $k, U=\left[U_{1}, \ldots, U_{n}\right]=\left[V_{1}, \ldots, V_{T}\right]^{\prime}$. For (2.1), $Y=\left[R_{1}, \ldots, R_{n}\right], X=\left[\iota_{T}, \tilde{R}_{\mathrm{M}}\right], R_{i}=\left(R_{i 1}, \ldots, R_{i T}\right)^{\prime}, \tilde{R}_{\mathrm{M}}=\left(\tilde{R}_{\mathrm{M} 1}, \ldots, \tilde{R}_{\mathrm{M} T}\right)^{\prime}$, $B=[a, \beta]^{\prime}, a=\left(a_{1}, \ldots, a_{n}\right)^{\prime}, \beta=\left(\beta_{1}, \ldots, \beta_{n}\right)^{\prime}$, and $\iota_{j}$ refers to a $j$-dimensional vector of ones (for any $j$ ). We shall also use the following equivalent forms for the model and hypotheses considered:

$$
\begin{gathered}
\tilde{Y}=Y-\tilde{R}_{\mathrm{M}} \iota_{n}^{\prime}=X C+U, \quad C=B-\Delta=\left[a, \beta-\iota_{n}\right]^{\prime}, \quad \Delta=\left[0, \iota_{n}\right]^{\prime}, \\
\tilde{\mathcal{H}}\left(\gamma_{0}\right): H\left(\gamma_{0}\right) C=0, \quad H\left(\gamma_{0}\right)=\left(1, \gamma_{0}\right) \quad \text { where } \gamma_{0} \text { is specified, } \\
\tilde{\mathcal{H}}_{\mathrm{B}}: H(\gamma) C=0, \quad H(\gamma)=(1, \gamma), \quad \text { for some } \gamma \in \Gamma .
\end{gathered}
$$

We further assume that we can condition on $\tilde{R}_{\mathrm{M}}$ and

$$
V_{t}=\left(u_{1 t}, \ldots, u_{n t}\right)^{\prime}=K^{\prime} W_{t}, \quad t=1, \ldots, T, \quad W_{t}=\left(W_{1 t}, \ldots, W_{n t}\right)^{\prime},
$$


where $K$ is unknown and nonsingular, $W=\left[W_{1}, \ldots, W_{T}\right]^{\prime}$ is independent of $X$, and the distribution of $W$ is either fully specified or specified up to an unknown distributional shape parameter $\nu$. We first present results which require no further regularity assumptions. We also consider further restrictions, which entail that the distribution of $W$ belongs to a specific family $\mathcal{H}_{W}(\mathcal{D}, \nu)$, where $\mathcal{D}$ represents a distribution type and $\nu \in \Omega_{\mathcal{D}}$ any (eventual) nuisance parameter characterizing the distribution. In particular, we consider the multivariate normal $\left(\mathcal{D}_{N}\right)$, Student- $\boldsymbol{t}\left(\mathcal{D}_{\boldsymbol{t}}\right)$ and normal mixture $\left(\mathcal{D}_{m}\right)$ distributions:

$$
\begin{gathered}
\mathcal{H}_{W}\left(\mathcal{D}_{N}\right): W_{t} \stackrel{i . i . d}{\sim} \mathrm{N}\left[0, I_{n}\right], \\
\mathcal{H}_{W}\left(\mathcal{D}_{\boldsymbol{t}}, \kappa\right): W_{t}=Z_{1 t} /\left(Z_{2 t} / \kappa\right)^{1 / 2}, Z_{1 t} \stackrel{\text { i.i.d }}{\sim} \mathrm{N}\left[0, I_{n}\right], Z_{2 t} \stackrel{i . i . d}{\sim} \chi^{2}(\kappa), \\
\mathcal{H}_{W}\left(\mathcal{D}_{m}, \pi, \omega\right): W_{t}=\mathrm{I}_{t}(\pi) Z_{1 t}+\left[1-\mathrm{I}_{t}(\pi)\right] Z_{3 t}, Z_{3 t} \stackrel{i . i . d}{\sim} \mathrm{N}\left[0, \omega I_{n}\right], 0<\pi<1,
\end{gathered}
$$

where $Z_{2 t}$ and $Z_{3 t}$ are independent of $Z_{1 t}$, and $\mathrm{I}_{t}(\pi)$ is an indicator random variable independent of $\left(Z_{1 t}, Z_{3 t}\right)$ such that $\mathrm{P}\left[\mathrm{I}_{t}(\pi)=0\right]=1-\mathrm{P}\left[\mathrm{I}_{t}(\pi)=1\right]=\pi$. So, in (2.8), $\nu=\kappa$ under (2.10), and $\nu=(\pi, \omega)$ under (2.11). If $E\left(W_{t} W_{t}^{\prime}\right)=I_{n}$, the covariance of $W_{t}$ is $\Sigma=K^{\prime} K$. $\Sigma$ is positive definite with further further restrictions. However, further constraints are needed in order for $K$ to be uniquely determined. If $W_{t}$ is Gaussian, we may assume that $K$ corresponds to the Cholesky factorization of $\Sigma$. Time-dependence may be fit via appropriate specifications for the distribution of $W_{t}, t=1, \ldots, T$. Since time varying volatility is prevalent in financial data, we consider the parametric GARCH structure:

$$
u_{i t}=w_{i t} h_{i t}^{\frac{1}{2}}, \quad h_{i t}=\left(1-\phi_{1 i}-\phi_{2 i}\right) \sigma_{i}^{2}+\phi_{1 i} w_{i, t-1}^{2}+\phi_{2 i} h_{i, t-1},
$$

where $w_{i t}$ are uncorrelated standard normal variables. This process may easily be reparametrized as in (2.8), where $K$ is a diagonal matrix with diagonal terms $\left(1-\phi_{1 i}-\phi_{2 i}\right)^{1 / 2} \sigma_{i}, i=1, \ldots, n$, and each $W_{i t}$ follows a univariate stationary GARCH process with unit intercept. Conforming with the above notation, we refer to this distributional hypothesis as $\mathcal{H}_{W}\left(\mathcal{D}_{G}, \phi\right)$, where $\phi$ is the $2 n \times 1$ vector $\left(\phi_{11}, \ldots, \phi_{1 n}, \phi_{21}, \ldots, \phi_{2 n}\right)^{7}$

Even though $a_{i}$ and $\beta_{i}$ are well identified, $\gamma$ is defined through a nonlinear transformation that may fail to be well-defined: the ratio $\gamma=a_{i} /\left(1-\beta_{i}\right)$ is not defined or, equivalently, the equation $a_{i}=\gamma\left(1-\beta_{i}\right)$ does not have a unique solution, when $\beta_{i}=1$. In such situations, the distributions of many standard test statistics become non-standard, so the corresponding tests are unreliable and the associated confidence sets invalid. In particular, asymptotic standard errors are unreliable measures of uncertainty and standard asymptotically justified $t$-type tests and confidence intervals have sizes that may deviate arbitrarily from their nominal levels; see the literature on weak identification [as reviewed, for example, in Dufour (2003) and Stock et al. (2002)]. Both the finite and large-sample distributional theory of most test statistics can be affected. While the discontinuity at $\beta_{i}=1$ is straightforward to see, the analysis below reveals that this is in fact not the whole story. In particular, we study the properties of estimators and test statistics following data transformations of the form $\tilde{Y}_{*}=\tilde{Y} A$, where $A$ is any nonsingular fixed matrix of order $n$. On comparing (2.1) to its transformed counterpart, we see that irregularities cannot be safely assumed away, even when

\footnotetext{
${ }^{7}$ Ideally, a multivariate GARCH structure may be considered if $T$ is sufficiently large relative to $n$; see Bauwens, Laurent and Rombouts (2006) for a recent survey. We adopt (2.12) since our empirical analysis relies on monthly data with 12 portfolios over 5 year subperiods (i.e. $T=60$ and $n=12$ ).
} 
observed betas are not close to one.

One of the most common inference methods in this context relies on the log-likelihood

$$
\ln [L(Y, B, \Sigma)]=-\frac{n T}{2}(2 \pi)-\frac{T}{2} \ln (|\Sigma|)-\frac{1}{2} \operatorname{tr}\left[\Sigma^{-1}(Y-X B)^{\prime}(Y-X B)\right] .
$$

The unrestricted MLE of $B$ and $\Sigma$ are:

$$
\hat{B}=\left(X^{\prime} X\right)^{-1} X^{\prime} Y=[\hat{a}, \hat{\beta}]^{\prime}, \quad \hat{\Sigma}=\hat{U}^{\prime} \hat{U} / T,
$$

where $\hat{U}=Y-X \hat{B}, \hat{a}=\left(\hat{a}_{1}, \ldots, \hat{a}_{n}\right)^{\prime}$ and $\hat{\beta}=\left(\hat{\beta}_{1}, \ldots, \hat{\beta}_{n}\right)^{\prime}$. The LR statistic to test $\mathcal{H}\left(\gamma_{0}\right)$ where $\hat{\Sigma}\left(\gamma_{0}\right)$ is the MLE of $\Sigma$ under $\mathcal{H}\left(\gamma_{0}\right)$ is:

$$
\begin{aligned}
L R\left(\gamma_{0}\right) & =T \ln \left[\Lambda\left(\gamma_{0}\right)\right], \quad \Lambda\left(\gamma_{0}\right)=\left|\hat{\Sigma}\left(\gamma_{0}\right)\right| /|\hat{\Sigma}|=\frac{n}{T-n-1} \mathcal{W}\left(\gamma_{0}\right)+1 \\
\hat{\Sigma}\left(\gamma_{0}\right) & =\hat{\Sigma}+\left(\hat{B}^{\prime} H\left(\gamma_{0}\right)^{\prime}\left[H\left(\gamma_{0}\right)\left(X^{\prime} X\right)^{-1} H\left(\gamma_{0}\right)^{\prime}\right]^{-1} H\left(\gamma_{0}\right) \hat{B}\right) / T \\
\mathcal{W}\left(\gamma_{0}\right) & =\frac{T-n-1}{n} \frac{\left(\hat{a}+\hat{\delta} \gamma_{0}\right)^{\prime} \hat{\Sigma}^{-1}\left(\hat{a}+\hat{\delta} \gamma_{0}\right)}{1+\left[\left(\hat{\mu}_{M}-\gamma_{0}\right)^{2} / \hat{\sigma}_{M}^{2}\right]}, \\
\hat{\mu}_{M} & =\frac{1}{T} \sum_{t=1}^{T} \tilde{R}_{\mathrm{M} t}, \quad \hat{\sigma}_{M}^{2}=\frac{1}{T} \sum_{t=1}^{T}\left(\tilde{R}_{\mathrm{M} t}-\hat{\mu}_{M}\right)^{2}, \quad \hat{\delta}=\hat{\beta}-\iota_{n} .
\end{aligned}
$$

$\mathcal{W}\left(\gamma_{0}\right)$ is the Hotelling statistic. Furthermore, the LR criterion to test $\mathcal{H}_{\mathrm{B}}$ is

$$
\begin{aligned}
L R_{\mathrm{B}} & =T \ln \left(\Lambda_{\mathrm{B}}\right)=\inf \left\{L R\left(\gamma_{0}\right): \gamma_{0} \in \Gamma\right\}=\operatorname{LR}(\hat{\gamma}) \\
\Lambda_{\mathrm{B}} & =\left|\hat{\Sigma}_{\mathrm{B}}\right| /|\hat{\Sigma}|, \quad\left|\hat{\Sigma}_{\mathrm{B}}\right|=\inf \left\{\left|\hat{\Sigma}\left(\gamma_{0}\right)\right|: \gamma_{0} \in \Gamma\right\}
\end{aligned}
$$

where $\hat{\Sigma}_{\mathrm{B}}$ is the MLE of $\Sigma$ under $\mathcal{H}_{\mathrm{B}}$ and $\hat{\gamma}$ is the unrestricted MLE of $\gamma$; see Shanken (1986). The log-likelihood for (2.5) is

$$
\ln [\tilde{L}(\tilde{Y}, C, \Sigma)]=\ln \left[L\left(Y-\tilde{R}_{\mathrm{M}} \iota_{n}^{\prime}, B-\Delta, \Sigma\right)\right]=\ln [L(Y, B, \Sigma)]
$$

and the LR statistics for testing $\tilde{\mathcal{H}}\left(\gamma_{0}\right)$ and $\tilde{\mathcal{H}}_{\mathrm{B}}$ coincide with $L R\left(\gamma_{0}\right)$ and $L R_{\mathrm{B}}$. If $\hat{C}$ is the MLE of $C$ in (2.5), GMM estimation leads to

$$
\hat{\vartheta}=\operatorname{vec}\left(\hat{C}^{\prime}\right)
$$

where for any $n \times k$ matrix $A$, $\operatorname{vec}(A)$ is the $(n k) \times 1$ vector obtained by stacking the columns of $A$ on top of each other. So $\mathcal{W}\left(\gamma_{0}\right)$ may be viewed as a Wald statistic based on the standardized distance between $\hat{a}+\hat{\delta} \gamma_{0}$ and zero, which conveys an asymptotic least-squares and a GMM interpretation of $\hat{\gamma}$. This may be exploited to allow for serial dependence, for example via a properly corrected weighting matrix. We consider the Wald-HAC statistic [see MacKinlay and Richardson (1991), Ravikumar et al. (2000), and Ray and Savin (2008)] where $\mathrm{R}=\left(1, \gamma_{0}\right) \otimes I_{n}$ and $\hat{U}_{t}^{\prime}$ is the $t$-th row 
of $\hat{U}$ :

$$
\mathcal{J}\left(\gamma_{0}\right)=T \hat{\vartheta}^{\prime} \mathrm{R}^{\prime}\left[\mathrm{R}\left(\left(\frac{X^{\prime} X}{T}\right)^{-1} \otimes I_{n}\right) \mathrm{S}_{T}\left(\left(\frac{X^{\prime} X}{T}\right)^{-1} \otimes I_{n}\right) \mathrm{R}^{\prime}\right]^{-1} \mathrm{R} \hat{\vartheta}
$$

where

$$
\mathrm{S}_{T}=\Psi_{0, t}+\sum_{j=1}^{q}\left(\frac{q-j}{q}\right)\left[\Psi_{j, T}+\Psi_{j, T}^{\prime}\right], \quad \Psi_{j, T}=\frac{1}{T} \sum_{t=j+1}^{T}\left(X_{t} \otimes \hat{U}_{t}\right)\left(X_{t-j} \otimes \hat{U}_{t-j}\right)^{\prime} .
$$

Under $\mathcal{H}\left(\gamma_{0}\right), \mathcal{J}\left(\gamma_{0}\right)$ follows a $\chi^{2}(n)$ distribution asymptotically. A GMM estimator $\tilde{\gamma}$ of $\gamma$ can be obtained by solving the problem

$$
\mathcal{J}_{\mathrm{B}}=\inf \left\{\mathcal{J}\left(\gamma_{0}\right): \gamma_{0} \in \Gamma\right\}=\mathcal{J}(\tilde{\gamma})
$$

A Wald-type formula for an asymptotic information-matrix-based standard error associated with $\hat{\gamma}$ is provided by Campbell et al. (1997, Chapter 5, equation 5.3.81):

$$
\operatorname{Var}(\hat{\gamma})=\frac{1}{T}\left(1+\frac{\left(\hat{\mu}_{M}-\gamma\right)^{2}}{\hat{\sigma}_{M}^{2}}\right)\left[\left(\iota_{n}-\beta\right)^{\prime} \Sigma^{-1}\left(\iota_{n}-\beta\right)\right]^{-1} .
$$

Whereas corrections may be derived for the non-Gaussian case [as in Barone-Adesi, Gagliardini and Urga (2004) who study a related asset pricing problem], the fact remains that (2.24) or regular "sandwich-type" corrections would depend non-trivially on $\gamma, \beta$ and particularly on $\Sigma$, leading to serious irregularities. For example, $\operatorname{Var}(\hat{\gamma})$ involves a division by $\left(\iota_{n}-\beta\right)^{\prime} \Sigma^{-1}\left(\iota_{n}-\beta\right)$ and thus becomes ill-defined at the unit beta boundary; this divisor also illustrates the role $\Sigma$ plays is determining the precision of $\hat{\gamma}$.

Throughout the paper, we use the following notation. We call $L R_{\mathrm{B}}$ and $L R\left(\gamma_{0}\right)$ quasi likelihood ratio (QLR) criteria and the associated MLEs quasi maximum likelihood (QML) estimators. We denote the observed value of these statistics as $L R_{\mathrm{B}}^{(0)}$ and $L R^{(0)}\left(\gamma_{0}\right)$, respectively. $\mathrm{P}_{(B, K)}$ represents the distribution of $Y$ when the parameters are $(B, K)$. For any matrix $A, M(A)=I-A\left(A^{\prime} A\right)^{-} A^{\prime}$.

\section{Identification-robust Monte Carlo tests for $\gamma$}

We will now derive the exact null distribution of the QLR statistic $L R\left(\gamma_{0}\right)$ under $\mathcal{H}\left(\gamma_{0}\right)$, where $\gamma_{0}$ is known. This will allow us to build a CS for $\gamma$ and yield a way of testing efficiency. The basic distributional result for that purpose is given by the following theorem.

Theorem 3.1 DistribUtion OF THE MEAN-VARIANCE CAPM TEST FOR A KNOWN ZEROBETA RATE. Under (2.1), (2.8) and $\mathcal{H}\left(\gamma_{0}\right), L R\left(\gamma_{0}\right)$ is distributed like

$$
\overline{L R}\left(\gamma_{0}, W\right)=T \ln \left(\left|W^{\prime} \bar{M}\left(\gamma_{0}\right) W\right| /\left|W^{\prime} M W\right|\right)
$$

where $\bar{M}\left(\gamma_{0}\right)=M(X)+X\left(X^{\prime} X\right)^{-1} H\left(\gamma_{0}\right)^{\prime}\left[H\left(\gamma_{0}\right)\left(X^{\prime} X\right)^{-1} H\left(\gamma_{0}\right)^{\prime}\right]^{-1} H\left(\gamma_{0}\right)\left(X^{\prime} X\right)^{-1} X^{\prime}$. 
Proofs are given in the Appendix. In the i.i.d. Gaussian case (2.9), we have:

$$
[(T-1-n) / n]\left[\Lambda\left(\gamma_{0}\right)-1\right] \sim F(n, T-1-n) ;
$$

see Dufour and Khalaf (2002). This result was used by Gibbons et al. (1989) in studying efficiency with an observable risk-free rate. Indeed, testing $\mathcal{H}\left(\gamma_{0}\right)$ is equivalent to testing whether the intercepts are jointly zero in a market model with returns in excess of $\gamma_{0}$.

For non-Gaussian distributions compatible with (2.8) [including the GARCH case (2.12)], Theorem 3.1 shows that the exact distribution of $L R\left(\gamma_{0}\right)$, although non-standard, may easily be simulated once $X$, the distribution of $W$ and $\gamma_{0}$ [given by $\mathcal{H}\left(\gamma_{0}\right)$ ] are set. So the Monte Carlo (MC) test method can be easily applied; see Dufour (2006). In general, this method assesses the rank of the observed value of a test statistic [denoted $S^{(0)}$ ], relative to a finite number $N$ of simulated statistics [denoted $S^{(1)}, \ldots, S^{(N)}$ ] drawn under the null hypothesis. Conforming with (2.8), we assume that $S^{(1)}, \ldots, S^{(N)}$ can be simulated given: (i) a value of $\nu$, (ii) $N$ draws $W^{(1)}, \ldots, W^{(N)}$ from the distribution of $W$ [which under (2.8) can be simulated once $\nu$ is specified], (iii) a vector of parameters (denoted $\eta$ ) which affects the distribution of the test statistic, and (iv) the test function $\bar{S}(\eta, W)$ which depends on $\eta, W$ and $X .^{8}$ In other words, on drawing $N$ samples from the distribution of $W$ (which may depend on $\nu$ ) and computing $\bar{S}(\eta, W)$ for each simulated sample, we get the vector $\bar{S}_{N}(\eta, \nu)=\left[\bar{S}\left(\eta, W^{(1)}\right), \ldots, \bar{S}\left(\eta, W^{(N)}\right)\right]^{\prime}$. In the case of $L R\left(\gamma_{0}\right), S^{(0)} \equiv L R^{(0)}\left(\gamma_{0}\right), \eta \equiv$ $\gamma_{0}$, and using (3.1), $\bar{S}\left(\eta, W^{(i)}\right)=\overline{L R}\left(\gamma_{0}, W^{(i)}\right)$. Given the above, a $M C p$-value is defined as:

$$
\begin{aligned}
p_{N}\left[S^{(0)} \mid \bar{S}_{N}(\eta, \nu)\right] & =\frac{N G_{N}\left[S^{(0)} ; \bar{S}_{N}(\eta, \nu)\right]+1}{N+1} \\
G_{N}\left[S^{(0)} ; \bar{S}_{N}(\eta, \nu)\right] & =\frac{1}{N} \sum_{j=1}^{N} I_{[0, \infty)}\left[\bar{S}\left(W^{(j)}, \eta\right)-S^{(0)}\right]
\end{aligned}
$$

where $I_{A}[x]=1$, if $x \in A$, and $I_{A}[x]=0$, if $x \notin A$. If the distribution of the statistic under consideration, given $X$, is completely determined by $X$ and the distribution of $W$ given $X$ (which depends on $\nu$ and $\eta$ ), then comparing $p_{N}\left[S^{(0)} \mid \bar{S}_{N}(\eta, \nu)\right]$ to an $\alpha$ cut-off where $\alpha(N+1)$ is an integer yields a test with the stated size $\alpha$ : the probability of rejection under the null hypothesis is exactly $\alpha$, for finite $T$ and $N$.

If $\nu$ or $\eta$ is not set by the null hypothesis, then the MMC method does allow one to control the level of the test: we maximize $p_{N}\left[S^{(0)} \mid \bar{S}_{N}(\eta, \nu)\right]$ over all the $(\nu, \eta)$ values compatible with the null hypothesis, and reject the latter if the maximal $p$-value is less than or equal to $\alpha$. Then the probability of rejection under the null hypothesis is itself not larger than $\alpha$, for finite $T$ and $N$; see Dufour (2006). In the case of $L R\left(\gamma_{0}\right)$ with $\overline{L R}_{N}\left(\gamma_{0}, \nu\right)=\left[\overline{L R}\left(\gamma_{0}, W^{(1)}\right), \ldots, \overline{L R}\left(\gamma_{0}, W^{(N)}\right)\right]^{\prime}$, we have:

$$
\hat{p}_{N}\left(\gamma_{0}, \nu\right) \equiv p_{N}\left[L R^{(0)}\left(\gamma_{0}\right) \mid \overline{L R}_{N}\left(\gamma_{0}, \nu\right)\right] .
$$

As a result of Theorem 3.1, we have, under $\mathcal{H}\left(\gamma_{0}\right)$ in conjunction with $\mathcal{H}_{W}(\mathcal{D}, \nu)$ :

$$
\begin{gathered}
\mathrm{P}\left[\hat{p}_{N}\left(\gamma_{0}, \nu_{0}\right) \leq \alpha\right]=\alpha, \text { when } \nu=\nu_{0}, \\
\mathrm{P}\left[\sup \left\{\hat{p}_{N}\left(\gamma_{0}, \nu\right): \nu \in \Omega_{\mathcal{D}}\right\} \leq \alpha\right] \leq \alpha, \text { when } \nu \text { may be unknown. }
\end{gathered}
$$

\footnotetext{
${ }^{8}$ For notational simplicity, the dependence upon $X$ is implicit through the definition of $\bar{S}$.
} 
We will call $\hat{p}_{N}\left(\gamma_{0}, \nu\right)$ a pivotal $M C(\mathrm{PMC}) p$-value.

\section{Identification-robust confidence sets for $\gamma$}

Under $\mathcal{H}_{\mathrm{B}}$, the ratios $a_{i} /\left(1-\beta_{i}\right), 1, \ldots, n$, are equal. This definition of $\gamma$ leads to the classical problem of inference on ratios from Fieller (1954). The problem here is clearly more complex, so to extend Fieller's arguments, we use the above defined tests of $\mathcal{H}\left(\gamma_{0}\right)$.

\subsection{Fieller-type confidence sets: the i.i.d. Gaussian case}

Consider the Gaussian model given by (2.1), (2.8) and (2.9). In this case, under $\mathcal{H}_{0}\left(\gamma_{0}\right), \mathcal{W}\left(\gamma_{0}\right)$ follows a Fisher distribution $F(n, T-n-1)$; see (3.2). Let $F_{\alpha}$ denote the cut-off point for a test with level $\alpha$ based on the $F(n, T-n-1)$ distribution. Then

$$
C F_{\gamma}(\alpha)=\left\{\gamma_{0} \in \Gamma: \mathcal{W}\left(\gamma_{0}\right) \leq F_{\alpha}\right\}
$$

has level $1-\alpha$ for $\gamma$, i.e. the probability that $\gamma$ be covered by $C F_{\gamma}(\alpha)$ is not smaller than $1-\alpha$ : Indeed, $\mathrm{P}\left[\gamma \in C F_{\gamma}(\alpha)\right]=1-\alpha$. On noting that $\mathcal{W}\left(\gamma_{0}\right) \leq F_{\alpha}$ can be rewritten as

$$
\begin{gathered}
M_{F}\left(\gamma_{0}\right)-\frac{n F_{\alpha}}{T-n-1} N_{F}\left(\gamma_{0}\right) \leq 0 \\
M_{F}\left(\gamma_{0}\right)=\left(\hat{a}+\hat{\delta} \gamma_{0}\right)^{\prime} \hat{\Sigma}^{-1}\left(\hat{a}+\hat{\delta} \gamma_{0}\right)=\left(\hat{\delta}^{\prime} \hat{\Sigma}^{-1} \hat{\delta}\right) \gamma_{0}^{2}+\left(2 \hat{\delta}^{\prime} \hat{\Sigma}^{-1} \hat{a}\right) \gamma_{0}+\hat{a}^{\prime} \hat{\Sigma}^{-1} \hat{a} \\
N_{F}\left(\gamma_{0}\right)=1+\frac{\left(\hat{\mu}_{M}-\gamma_{0}\right)^{2}}{\hat{\sigma}_{M}^{2}}=\frac{1}{\hat{\sigma}_{M}^{2}} \gamma_{0}^{2}-\frac{2 \hat{\mu}_{m}}{\hat{\sigma}_{M}^{2}} \gamma_{0}+1+\frac{\hat{\mu}_{M}^{2}}{\hat{\sigma}_{M}^{2}}
\end{gathered}
$$

we see, after a few manipulations, that $C F_{\gamma}(\alpha)$ reduces to a simple quadratic inequation:

$$
\begin{gathered}
C F_{\gamma}(\alpha)=\left\{\gamma_{0} \in \Gamma: A \gamma_{0}^{2}+B \gamma_{0}+C \leq 0\right\}, \\
A=\hat{\delta}^{\prime} \hat{\Sigma}^{-1} \hat{\delta}-\left(\frac{n F_{\alpha}}{T-n-1}\right) \frac{1}{\hat{\sigma}_{M}^{2}}, \quad B=2\left[\hat{\delta}^{\prime} \hat{\Sigma}^{-1} \hat{a}+\left(\frac{n F_{\alpha}}{T-n-1}\right) \frac{\hat{\mu}_{M}}{\hat{\sigma}_{M}^{2}}\right], \\
C=\hat{a}^{\prime} \hat{\Sigma}^{-1} \hat{a}-\left(\frac{n F_{\alpha}}{T-n-1}\right)\left[1+\frac{\hat{\mu}_{M}^{2}}{\hat{\sigma}_{M}^{2}}\right] .
\end{gathered}
$$

For $\Gamma=\mathbb{R}$, the resulting CS can take several forms depending on the roots of the polynomial $A \gamma_{0}^{2}+B \gamma_{0}+C$ : (a) a closed interval; (b) the union of two unbounded intervals; (c) the entire real line; (d) an empty set. ${ }^{9}$ Case (a) corresponds to a situation where $\gamma$ is well identified, while (b) and (c) correspond to unbounded CSs and indicate (partial or complete) non-identification. The possibility of getting an empty CS may appear surprising. But, on hindsight, this is quite natural: it means that no value of $\gamma_{0}$ does allow $\mathcal{H}\left(\gamma_{0}\right)$ to be acceptable. Since $\mathcal{H}_{\mathrm{B}}$ states that there exists a real scalar $\gamma$ such that $a_{i}=\left(1-\beta_{i}\right) \gamma, i=1, \ldots, n$, this can be interpreted as a rejection of $\mathcal{H}_{\mathrm{B}}$. Further, under $\mathcal{H}_{\mathrm{B}}$, the probability that $C F_{\gamma}(\alpha)$ covers the true value $\gamma$ is $1-\alpha$, and an empty set obviously does not cover $\gamma$. Consequently, the probability that $C F_{\gamma}(\alpha)$ be empty $\left[C F_{\gamma}(\alpha)=\emptyset\right]$

\footnotetext{
${ }^{9}$ For further discussion, see Dufour and Jasiak (2001), Zivot et al. (1998), Dufour and Taamouti (2005), Kleibergen (2009), and Mikusheva (2009).
} 
cannot be greater than $\alpha$ under $\mathcal{H}_{\mathrm{B}}: \mathrm{P}\left[C F_{\gamma}(\alpha)=\emptyset\right] \leq \alpha$. The event $C F_{\gamma}(\alpha)=\emptyset$, is a test with level $\alpha$ for $\mathcal{H}_{\mathrm{B}}$ under normality.

\subsection{Fieller-type confidence sets with non-Gaussian non-i.i.d. errors}

The quadratic CS described above relies heavily on the fact that the same critical point $F_{\alpha}$ can be used to test all values of $\gamma_{0}$. This occurs under the i.i.d. Gaussian distributional assumption, but not necessarily otherwise. Although the quadratic CS will remain "asymptotically valid" as long as $\mathcal{W}\left(\gamma_{0}\right)$ converges to a $\chi^{2}(n)$ distribution, this cannot provide an exact CS. The Fiellertype procedure can be extended to allow for possibly non-Gaussian disturbances, by inverting an $\alpha$-level test based on $\mathcal{W}\left(\gamma_{0}\right)$ [or equivalently on $L R\left(\gamma_{0}\right)$ ] performed by simulation (as a MC test). Consider the MC $p$-value $\hat{p}_{N}\left(\gamma_{0}, \nu\right)$ function associated with this statistic, as defined in (3.5). Since the critical region $\hat{p}_{N}\left(\gamma_{0}, \nu\right) \leq \alpha$ has level $\alpha$ for testing $\gamma=\gamma_{0}$ when $\nu$ is known, the set of $\gamma_{0}$ values for which $\hat{p}_{N}\left(\gamma_{0}, \nu\right)$ exceeds $\alpha$, i.e.

$$
C_{\gamma}(\alpha ; \nu)=\left\{\gamma_{0} \in \Gamma: \hat{p}_{N}\left(\gamma_{0}, \nu\right)>\alpha\right\}
$$

is a CS with level $1-\alpha$ for $\gamma$. Similarly, when $\nu$ is not specified, the test $\sup \left\{p_{N}\left(\gamma_{0}, \nu_{0}\right): \nu_{0} \in\right.$ $\left.\Omega_{\mathcal{D}}\right\} \leq \alpha$ yields:

$$
C_{\gamma}(\alpha ; \mathcal{D})=\left\{\gamma_{0} \in \Gamma: \sup \left\{\hat{p}_{N}\left(\gamma_{0}, \nu_{0}\right): \nu_{0} \in \Omega_{\mathcal{D}}\right\}>\alpha\right\},
$$

whose level is also $1-\alpha . C_{\gamma}(\alpha ; \nu)$ or $C_{\gamma}(\alpha ; \mathcal{D})$ must be drawn by numerical methods. Our empirical analysis reported below, relies on nested grid searches, over $\gamma_{0}$ and $\kappa$, for the Student- $t$ case (2.10), and over $\gamma_{0}$ and $(\pi, \omega)$ for the normal-mixture case (2.11); for the GARCH case (2.12), we conduct a grid search on $\gamma_{0}$ where for each candidate value, we run the simulated annealing optimization algorithm to calculate the maximal $p$-value from (4.9) over the $2 n$ nuisance parameters in $\phi$.

We have no closed-form description of the structure of $C_{\gamma}(\alpha ; \nu)$ or $C_{\gamma}(\alpha ; \mathcal{D})$. While these can be bounded intervals (this is showed numerically in Section 8), $C_{\gamma}(\alpha ; \nu)$ or $C_{\gamma}(\alpha ; \mathcal{D})$ must be unbounded with a high probability if $\gamma$ is not identifiable or weakly identified [see Dufour (1997)]. An empty CS is also possible and provides evidence that $\mathcal{H}_{\mathrm{B}}$ is not compatible with the data. The event $C_{\gamma}(\alpha ; \nu)=\emptyset$ [or $C_{\gamma}(\alpha ; \mathcal{D})=\emptyset$ ] is a test with level $\alpha$ for $\mathcal{H}_{\mathrm{B}}$ under (2.8). The identity $L R(\hat{\gamma})=\inf \left\{L R\left(\gamma_{0}\right): \gamma_{0} \in \Gamma\right\}$ entails that $\hat{\gamma}$ must belong to the CS, provided its level is $>0$.

The Hotelling-based CS we obtain for the GARCH case is exact, because the cut-off point we use when inverting $\mathcal{W}\left(\gamma_{0}\right)$ is adjusted for the parametric form (2.12) via the maximized $p$-value from (4.9). Inverting $\mathcal{J}\left(\gamma_{0}\right)$ in (2.22) may however be more appropriate. Again, this must be implemented by numerical methods; for example, a grid search can be conducted on $\gamma_{0}$ where for each candidate value, $\mathcal{J}\left(\gamma_{0}\right)$ is referred to the $\chi^{2}(n)$ distribution; this would circumvent the identification problem asymptotically [as argued e.g. in Stock and Wright (2000)], yet in finite samples, the $\chi^{2}(n)$ approximation may perform poorly. Indeed, our simulation results reported below illustrate the severity of this problem. Consequently, we use the MMC method for each candidate $\gamma_{0}$ : we maximize over the model parameters as well as over $\phi .^{10}$

\footnotetext{
${ }^{10}$ We have observed a numerical invariance to $B$ and $K$, which calls for further theoretical work with such statistics; see also Section 7.
} 


\section{Invariance and exact distribution of $L R_{\mathrm{B}}$}

In this section, we study the exact distribution of the statistics $L R\left(\gamma_{0}\right)$ and $L R_{\mathrm{B}}$, under both the null hypothesis and the corresponding unrestricted MLR alternative model. We track and control for the joint role betas and scale parameters play in identifying $\gamma$.

Lemma 5.1 MUlTIVARIATE SCALE INVARIANCE. The LR statistics $L R\left(\gamma_{0}\right)$ and $L R_{\mathrm{B}}$ defined in (2.18) and (2.14) are invariant to replacing $\tilde{Y}$ by $\tilde{Y}_{*}=\tilde{Y} A$, where $A$ is an arbitrary nonsingular $n \times n$ matrix.

Such transformations can be viewed as the following affine transformations on $Y$ :

$$
Y_{*}=Y A+\tilde{R}_{\mathrm{M}} \iota_{n}^{\prime}\left(I_{n}-A\right) .
$$

Theorem 5.2 EXACT DISTRIBUTION OF BCAPM LR TESTS. Under (2.1) and (2.8), the distributions of $L R\left(\gamma_{0}\right)$ and $L R_{\mathrm{B}}$ depend on $(B, K)$ only through $\bar{B}=(B-\Delta) K^{-1}$, and

$$
L R\left(\gamma_{0}\right)=T \ln \left(\left|\hat{W}\left(\gamma_{0}\right)^{\prime} \hat{W}\left(\gamma_{0}\right)\right| /\left|\hat{W}^{\prime} \hat{W}\right|\right), \quad L R_{\mathrm{B}}=\inf \left\{L R\left(\gamma_{0}\right): \gamma_{0} \in \Gamma\right\}
$$

where $\Delta=\left[0, \iota_{n}\right]^{\prime}, \hat{W}=M(X) W, \bar{M}\left(\gamma_{0}\right)$ is defined as in (3.1) and

$$
\hat{W}\left(\gamma_{0}\right)=\bar{M}\left(\gamma_{0}\right)(X \bar{B}+W)=\bar{M}\left(\gamma_{0}\right)\left\{\iota_{T}\left[a+\gamma_{0}\left(\beta-\iota_{n}\right)\right]^{\prime} K^{-1}+W\right\} .
$$

If, furthermore, the null hypothesis $\mathcal{H}_{\mathrm{B}}$ holds, then

$$
\hat{W}\left(\gamma_{0}\right)=\left(\gamma_{0}-\gamma\right) \bar{M}\left(\gamma_{0}\right) \iota_{T}\left(\beta-\iota_{n}\right)^{\prime} K^{-1}+\bar{M}\left(\gamma_{0}\right) W
$$

and the distribution of $L R_{\mathrm{B}}$ depends on $(B, K)$ only through $\gamma$ and $\left(\beta-\iota_{n}\right)^{\prime} K^{-1}$; in the Gaussian case (2.9), this distribution involves only one nuisance parameter.

Even though $B$ and $K$ may involve up to $2 n+n^{2}$ different nuisance parameters [or $2 n+n(n+$ 1) $/ 2$ parameters, if $K$ is triangular], the latter theorem shows that the number of free parameters in the distributions of $L R\left(\gamma_{0}\right)$ and $L R_{\mathrm{B}}$ does not exceed $2 n$; when $\mathcal{H}_{\mathrm{B}}$ holds, the number of free parameters is at most $n+1$. Further, under $\mathcal{H}\left(\gamma_{0}\right)$ [using (5.4)] $\bar{B}$ is evacuated, entailing Theorem 3.1. Theorem 5.2 also provides the power function.

\section{Exact bound procedures for testing $\mathcal{H}_{\mathrm{B}}$}

In this section, we propose tests for $\mathcal{H}_{\mathrm{B}}$ in the presence of nuisance parameters induced by nonlinearity and non-Gaussian error distributions. We study first global bounds based on tests of $\mathcal{H}\left(\gamma_{0}\right)$ where we outline important differences between the Gaussian and non-Gaussian cases. Second, we describe more general but computationally more expensive methods based on the technique of MMC tests to obtain tighter bounds.

\subsection{Global bound induced by tests of $\mathcal{H}\left(\gamma_{0}\right)$}

The results of Section 3 on testing $\gamma=\gamma_{0}$ can be used to derive a global bound on the distribution of the statistic $L R_{\mathrm{B}}$. This is done in the following theorem. 
Theorem 6.1 Global bOUND ON THE NUll Distribution of THE BCAPM TEST. Under the assumptions $(2.1),(2.8)$ and $\mathcal{H}_{\mathrm{B}}$, we have, for any given $\nu \in \Omega_{\mathcal{D}}$,

$$
\mathrm{P}\left[L R_{\mathrm{B}} \geq x\right] \leq \sup _{\gamma_{0} \in \Gamma} \mathrm{P}\left[\overline{L R}\left(\gamma_{0}, W\right) \geq x\right], \forall x,
$$

where $\overline{L R}\left(\gamma_{0}, W\right)$ is defined in (3.1). Further, in the Gaussian case (2.9), we have:

$$
\mathrm{P}\left[(T-1-n)\left(\Lambda_{\mathrm{B}}-1\right) / n \geq x\right] \leq \mathrm{P}[F(n, T-1-n) \geq x], \forall x .
$$

To relate this result to available bounds, observe that (6.1) and (6.2) easily extend to the following multi-beta setups: for $i=1, \ldots, n, t=1, \ldots, T$,

$$
R_{i t}=a_{i}+\sum_{j=1}^{s} \beta_{i j} \tilde{R}_{j t}+u_{i t}, \quad \mathcal{H}_{\mathrm{B}}: a_{i}=\gamma\left(1-\sum_{j=1}^{s} \beta_{i j}\right)
$$

where $\tilde{R}_{j t}, j=1, \ldots, s$, are returns on $s$ benchmarks. In this case, the bounding distribution of $L R_{\mathrm{B}}$ obtains as in Theorem 6.1 where $X=\left[\iota_{T}, \tilde{R}_{1}, \ldots, \tilde{R}_{s}\right], \tilde{R}_{j}=\left(\tilde{R}_{j 1}, \ldots, \tilde{R}_{j T}\right)^{\prime}$, $j=1, \ldots, s$, and $H$ is the $k$-dimensional row vector $\left(1, \gamma_{0}, \ldots, \gamma_{0}\right)$. In the Gaussian case, $\mathrm{P}\left[\overline{L R}\left(\gamma_{0}, W\right) \geq x\right]$ does not depend on $\gamma_{0}$, and the bounding distribution under normality is $F(n, T-s-n)$. Shanken (1986) suggested the statistic

$$
\hat{Q}=\min _{\gamma}\left\{\frac{T\left[\hat{a}-\gamma\left(\iota_{n}-\hat{\beta} \iota_{s}\right)\right]^{\prime}[(T /(T-2)) \hat{\Sigma}]^{-1}\left[\hat{a}-\gamma\left(\iota_{n}-\hat{\beta} \iota_{s}\right)\right]}{1+\left(\bar{R}_{\mathbf{M}}-\gamma \iota_{s}\right)^{\prime} \hat{\Delta}_{\mathbf{M}}^{-1}\left(\bar{R}_{\mathbf{M}}-\gamma \iota_{s}\right)}\right\}
$$

where $\hat{a}$ is an $n$-dimensional vector which includes the (unconstrained) intercept estimates, $\hat{\beta}$ is an $n \times s$ matrix whose rows include the unconstrained OLS estimates of $\left(\beta_{i 1}, \ldots, \beta_{i s}\right), i=$ $1, \ldots, n, \bar{R}_{\mathrm{M}}$ and $\hat{\Delta}_{\mathrm{M}}$ include respectively the time-series means and sample covariance matrix corresponding to the right-hand-side total portfolio returns. Further, the minimum in (6.4) occurs at the constrained MLE $\hat{\gamma}$ of $\gamma$, and

$$
L R_{\mathrm{B}}=T \ln (1+\hat{Q} /(T-s-1)) .
$$

For normal errors, $(T-s-n) \hat{Q} /[n(T-s-1)]$ can be bounded by the $F(n, T-n-s)$ distribution. The latter obtains from Gibbons et al.'s (1989) joint test of zero intercepts, where returns are expressed in excess of a known $\gamma$.

Independently, Stewart (1997) showed [using Dufour (1989)] that, under normal errors, (T $s-n)\left[\left(\left|\hat{\Sigma}_{\mathrm{B}}\right| /|\hat{\Sigma}|\right)-1\right] / n$ can be bounded by the $F(n, T-n-s)$ distribution. Now, from (2.18) and (6.5), we see that Shanken and Stewart's bounds are equivalent, and both results obtain from Theorem 6.1 in the special case of normal errors.

When disturbances are non-Gaussian, Theorem 6.1 entails that the bounding distribution can easily be simulated, as follows. Given a value of $\nu$, generate $N$ i.i.d. draws from the distribution of $W_{1}, \ldots, W_{T}$; then, for any given $\gamma_{0}$, these yield a vector $\overline{L R}_{N}\left(\gamma_{0}, \nu\right)$ of $N$ simulated values of the test statistic $\overline{L R}\left(\gamma_{0}, W\right)$, as defined in (3.1). A MC $p$-value may then be computed from the rank of the observed statistic $L R_{\mathrm{B}}$ relative to the simulated values. Denote this MC $p$-value by

$$
\hat{p}_{N}^{U}\left(\gamma_{0}, \nu\right) \equiv p_{N}\left[L R_{\mathrm{B}}^{(0)} \mid \overline{L R}_{N}\left(\gamma_{0}, \nu\right)\right]
$$


where $L R_{\mathrm{B}}^{(0)}$ represents the value of the test statistic $L R_{\mathrm{B}}$ based on the observed data; we will call $\hat{p}_{N}^{U}\left(\gamma_{0}, \nu\right)$ the bound MC (BMC) $p$-value. In contrast with the Gaussian case, $\hat{p}_{N}^{U}\left[\gamma_{0}, \nu\right]$ may depend on $\gamma_{0}$; nevertheless, for any $\gamma_{0}$,

$$
L R_{\mathrm{B}} \leq L R\left(\gamma_{0}\right) \Rightarrow \hat{p}_{N}\left(\gamma_{0}, \nu\right) \leq \hat{p}_{N}^{U}\left(\gamma_{0}, \nu\right) .
$$

So a critical region that provably satisfies the level constraint can be obtained by maximizing $\hat{p}_{N}^{U}\left(\gamma_{0}, \nu\right)$ over the relevant nuisance parameters. To simplify presentation of this result, we introduce the following notation. For any subsets $A \subseteq \Gamma$ and $E \subseteq \Omega_{\mathcal{D}}$, let

$$
\begin{gathered}
\hat{p}_{N}^{U}\left(\gamma_{0}, E\right)=\sup \left\{\hat{p}_{N}^{U}\left(\gamma_{0}, \nu_{0}\right): \nu_{0} \in E\right\}, \quad \hat{p}_{N}^{U}\left(A, \nu_{0}\right)=\sup \left\{\hat{p}_{N}^{U}\left(\gamma_{0}, \nu_{0}\right): \gamma_{0} \in A\right\}, \\
\hat{p}_{N}^{U}(A, E)=\sup \left\{\hat{p}_{N}^{U}\left(\gamma_{0}, \nu_{0}\right): \gamma_{0} \in A, \nu_{0} \in E\right\},
\end{gathered}
$$

where, by convention, $\hat{p}_{N}^{U}(A, \cdot)=0$ if $A$ is empty, and $\hat{p}_{N}^{U}(\cdot, E)=0$ if $E$ is empty.

Theorem 6.2 GLOBAL SIMULATION-BASED BOUND ON THE NULL DISTRIBUTION OF THE BCAPM TEST STATISTIC. Under $(2.1),(2.8)$ and $\mathcal{H}_{\mathrm{B}}$, we have:

$$
\mathrm{P}\left[\hat{p}_{N}^{U}(\Gamma, \nu) \leq \alpha\right] \leq \alpha, \quad \mathrm{P}\left[\hat{p}_{N}^{U}\left(\Gamma, \Omega_{\mathcal{D}}\right) \leq \alpha\right] \leq \alpha,
$$

where $\nu$ represents the true distributional shape of $W$.

The first inequality in (6.10) holds for a statistic that requires the value of $\nu$, while the second one holds even without the need to specify $\nu$. These bound tests are closely related to the CSbased test proposed in Section 4: the null hypothesis is rejected when the CS for $\gamma$ is empty, i.e. if no value of $\gamma_{0}$ can be deemed acceptable (at level $\alpha$ ), either with $\nu$ specified or $\nu$ taken as a nuisance parameter. This may be seen on comparing (4.9) with the probabilities in Theorem 6.2. On recalling that $L R_{\mathrm{B}}=\inf \left\{L R\left(\gamma_{0}\right): \gamma_{0} \in \Gamma\right\}$, the latter also suggests a relatively easy way of showing that $C_{\gamma}(\alpha ; \nu)$ or $C_{\gamma}(\alpha ; \mathcal{D})$ is not empty, through the specific $p$-value $\hat{p}_{N}^{U}(\hat{\gamma}, \nu)$ obtained by taking $\gamma_{0}=\hat{\gamma}$ in (6.6). We shall call $\hat{p}_{N}^{U}(\hat{\gamma}, \nu)$ the $Q M L$-BMC $p$-value.

Theorem 6.3 RELATION BETWEEN EFFICIENCY TESTS AND ZERO-BETA CONFIDENCE SETS. Under (2.1), (2.8) and $\mathcal{H}_{\mathrm{B}}$, let $\hat{\gamma}$ be the QML estimator of $\gamma$ in (2.19). Then,

$$
\begin{gathered}
\hat{p}_{N}^{U}(\hat{\gamma}, \nu)>\alpha \Rightarrow \sup \left\{\hat{p}_{N}\left(\gamma_{0}, \nu\right): \gamma_{0} \in \Gamma\right\}>\alpha \Rightarrow C_{\gamma}(\alpha ; \nu) \neq \emptyset, \forall \nu \in \Omega_{\mathcal{D}}, \\
\hat{p}_{N}^{U}\left(\hat{\gamma}, \Omega_{\mathcal{D}}\right)>\alpha \Rightarrow \sup \left\{\hat{p}_{N}\left(\gamma_{0}, \nu_{0}\right): \gamma_{0} \in \Gamma, \nu_{0} \in \Omega_{\mathcal{D}}\right\}>\alpha \Rightarrow C_{\gamma}(\alpha ; \mathcal{D}) \neq \emptyset,
\end{gathered}
$$

where $C_{\gamma}(\alpha, \nu)$ and $C_{\gamma}(\alpha ; \mathcal{D})$ are the sets defined in (4.8) and (4.9).

For the Gaussian case, Zhou (1991) and Velu and Zhou (1999) proposed a potentially tighter bound applicable to statistics which can be written as ratios of independent Wishart variables and does not seem to extend easily to other classes of distributions. In the next section, we propose an approach which yields similarly tighter bounds for non-Gaussian distributions as well. Finally, the HAC statistic $\mathcal{J}_{\mathrm{B}}$ may be used to obtain alternative identification-robust bound tests following the same rationale. The correspondence between such tests and empty CSs entailed by test inversion also follows from similar arguments. Finite-sample MMC level corrections are recommended, given the simulation results in Section 7. 


\subsection{Maximized Monte Carlo bounds}

Another approach to testing $\mathcal{H}_{\mathrm{B}}$ with the statistic $L R_{\mathrm{B}}$ consists in directly assessing its dependence on nuisance parameters and adjusting the test accordingly through the MMC method [Dufour (2006)]. Let $\theta=\psi(B, K)$ represent the parameter vector upon which the distribution of $L R_{\mathrm{B}}$ actually depends, and $\Omega_{\mathrm{B}}$ the set of admissible values for $\theta$ under $\mathcal{H}_{\mathrm{B}}$. The dimension of $\theta$ may be lower than than the number of parameters in $B$ and $K$. To conform with our earlier notation for MC $p$-values, we define the function $\overline{L R}_{\mathrm{B}}(\theta, W)=\overline{L R}_{\mathrm{B}}(\psi(B, K), W)$ which assigns to each value of $(B, K)$ and the noise matrix $W$ the following outcome: using $\theta$ and a draw from the distribution of $W$ (which may depend on $\nu$ ), generate a sample from (2.1)-(2.2), and compute $L R_{\mathrm{B}}$ [as defined in (2.18)] from this sample.

On applying $\overline{L R}_{\mathrm{B}}(\theta, W)$, we can get simulated values from the null distribution of $L R_{\mathrm{B}}$ for any value of $\theta$. If $N$ independent replications $W^{(1)}, \ldots, W^{(N)}$ of $W$ are generated, we can then compute the corresponding vector of $\overline{L R}_{\mathrm{B}}$ statistics and the $p$-value function $\hat{p}_{\mathrm{B} N}(\theta, \nu)=$ $p_{N}\left[L R_{\mathrm{B}}^{(0)} \mid \overline{L R}_{\mathrm{B} N}(\theta, \nu)\right]$, where $\overline{L R}_{\mathrm{B} N}(\theta, \nu)=\left[\overline{L R}_{\mathrm{B}}\left(\theta, W^{(1)}\right), \ldots, \overline{L R}_{\mathrm{B}}\left(\theta, W^{(N)}\right)\right]^{\prime}$. For any given value of $\nu$, the MMC $p$-value associated with $L R_{\mathrm{B}}^{(0)}$ is obtained by maximizing $\hat{p}_{\mathrm{B} N}(\theta, \nu)$ with respect to $\theta$ over the set of admissible values $\Omega_{\mathrm{B}}$ under $\mathcal{H}_{\mathrm{B}}$ :

$$
\hat{p}_{\mathrm{B} N}^{M}\left(\Omega_{\mathrm{B}}, \nu\right)=\sup \left\{\hat{p}_{\mathrm{B} N}(\theta, \nu): \theta \in \Omega_{\mathrm{B}}\right\} .
$$

Then, under $\mathcal{H}_{\mathrm{B}}$ and the error distribution associated with $\nu$, we have: $\mathrm{P}\left[\hat{p}_{\mathrm{B} N}^{M}\left(\Omega_{\mathrm{B}}, \nu\right) \leq \alpha\right] \leq \alpha$; see Dufour (2006). In other words, $\hat{p}_{\mathrm{B} N}^{M}\left(\Omega_{\mathrm{B}}, \nu\right) \leq \alpha$ is a critical region with level $\alpha$. Further, in order to allow for an unknown $\nu$, we can maximize $\hat{p}_{\mathrm{B} N}(\theta, \nu)$ with respect to $\nu \in \Gamma_{\mathcal{D}}$. Set: $\hat{p}_{\mathrm{B} N}^{M}\left(\theta, \Omega_{\mathcal{D}}\right)=\sup \left\{\hat{p}_{\mathrm{B} N}(\theta, \nu): \nu \in \Omega_{\mathcal{D}}\right\}, \hat{p}_{\mathrm{B} N}^{M}\left(\Omega_{\mathrm{B}}, \Omega_{\mathcal{D}}\right)=\sup \left\{\hat{p}_{\mathrm{B} N}^{M}\left(\theta, \Omega_{\mathcal{D}}\right): \theta \in \Omega_{\mathrm{B}}\right\}$. Then, under $\mathcal{H}_{\mathrm{B}}, \mathrm{P}\left[\hat{p}_{\mathrm{B} N}^{M}\left(\Omega_{\mathrm{B}}, \Omega_{\mathcal{D}}\right) \leq \alpha\right] \leq \alpha$.

Theorem 6.3 guarantees that $\hat{p}_{N}^{U}(\Gamma, \nu) \leq \alpha \Rightarrow \hat{p}_{\mathrm{B} N}^{M}\left(\Omega_{\mathrm{B}}, \nu\right) \leq \alpha$ for any given $\nu$. So it may be useful to check the global bound for significance before turning to the MMC one. Furthermore, it is not always necessary to run the numerical maximization underlying MMC to convergence: if $\hat{p}_{\mathrm{B} N}(\theta, \nu)>\alpha$ given any relevant $\theta$ (or $\nu$ ), then a non-rejection is confirmed. We suggest to use the QML estimate $\hat{\theta}$ of $\theta$ as start-up value, because this provides parametric bootstrap-type [or a local MC (LMC)] $p$-values:

$$
p_{N}^{b}(\nu)=\hat{p}_{\mathrm{B} N}(\hat{\theta}, \nu), p_{N}^{b}\left(\Omega_{\mathcal{D}}\right)=\hat{p}_{\mathrm{B} N}\left(\hat{\theta}, \Omega_{\mathcal{D}}\right) .
$$

Then $p_{N}^{b}(\nu)>\alpha$ entails $\hat{p}_{\mathrm{B} N}^{M}\left(\Omega_{\mathrm{B}}, \nu\right)>\alpha$, and $p_{N}^{b}\left(\Omega_{\mathcal{D}}\right)>\alpha$ entails $\hat{p}_{\mathrm{B} N}^{M}\left(\Omega_{\mathrm{B}}, \Omega_{\mathcal{D}}\right)>\alpha$.

Finally, a parametric MMC test imposing (2.12) may be applied to the HAC statistics $\mathcal{J}\left(\gamma_{0}\right)$ and $\mathcal{J}_{\mathrm{B}}$, as an attempt to correct their size for the GARCH alternative of interest. We investigate the size-corrected power associated with these statistics in Section 7.

\subsection{Two-stage bound confidence procedures}

To deal with the fact that the distribution of $W$ may involve an unknown parameter $\nu \in \Omega_{\mathcal{D}}$, we suggested above to maximize the relevant $p$-values over $\Omega_{\mathcal{D}}$. We next consider restricting the maximization over $\nu$ to a set which is empirically relevant, as in Beaulieu et al. (2007). This leads to two basic steps: (i) an exact CS with level $1-\alpha_{1}$ is built for $\nu$, and (ii) the MC $p$-values (presented above) are maximized over all values of $\nu$ in the latter CS and are referred to the level $\alpha_{2}$, so that 
the global test level is $\alpha=\alpha_{1}+\alpha_{2}$. In our empirical application, we used $\alpha / 2$. Let $\mathcal{C}_{\nu}\left(\alpha_{1}\right)=$ $\mathcal{C}_{\nu}\left(\alpha_{1} ; Y\right)$ be a CS with level $1-\alpha_{1}$ for $\nu$. Then, under $\mathcal{H}\left(\gamma_{0}\right)$, we have $\mathrm{P}\left[\hat{p}_{N}^{U}\left[\gamma_{0}, \mathcal{C}_{\nu}\left(\alpha_{1}\right)\right] \leq\right.$ $\left.\alpha_{2}\right] \leq \alpha_{1}+\alpha_{2}$ while, under $\mathcal{H}_{\mathrm{B}}$ :

$$
\mathrm{P}\left[\hat{p}_{N}^{U}\left[\Gamma, \mathcal{C}_{\nu}\left(\alpha_{1}\right)\right] \leq \alpha_{2}\right] \leq \alpha_{1}+\alpha_{2}, \quad \mathrm{P}\left[\hat{p}_{\mathrm{B} N}^{M}\left[\Omega_{\mathrm{B}}, \mathcal{C}_{\nu}\left(\alpha_{1}\right)\right] \leq \alpha_{2}\right] \leq \alpha_{1}+\alpha_{2} .
$$

Note also that for $\hat{p}_{\mathrm{BN}}^{M}\left[\Omega_{\mathrm{B}}, \mathcal{C}_{\nu}\left(\alpha_{1}\right)\right] \leq \alpha_{2}$ not to hold, the following condition is sufficient:

$$
\hat{p}_{\mathrm{BN}}^{M}\left(\hat{\theta}, \mathcal{C}_{\nu}\left(\alpha_{1}\right)\right)>\alpha_{2} .
$$

To build a CSs for $\nu$, we invert a test (of level $\alpha_{1}$ ) for the specification underlying (2.8) where $\nu=\nu_{0}$ for known $\nu_{0}$; this avoids the need to use regularity assumptions on $\nu$. The test we invert is the three-stage MC GF test introduced in Dufour et al. (2003):

$$
\mathrm{CSK}=1-\min \left\{\hat{p}\left[\operatorname{ESK}\left(\nu_{0}\right)\right], \hat{p}\left[\operatorname{EKU}\left(\nu_{0}\right)\right]\right\}
$$

where $\operatorname{ESK}\left(\nu_{0}\right)=\left|\mathrm{SK}-\overline{\mathrm{SK}}\left(\nu_{0}\right)\right|, \mathrm{SK}=\frac{1}{T^{2}} \sum_{t=1}^{T} \sum_{i=1}^{T} \hat{d}_{i t}^{3}, \operatorname{EKU}\left(\nu_{0}\right)=\left|\mathrm{KU}-\overline{\mathrm{KU}}\left(\nu_{0}\right)\right|$, $\mathrm{KU}=\frac{1}{T} \sum_{t=1}^{T} \hat{d}_{t t}^{2}, \hat{d}_{i t}$ are the elements of the matrix $\hat{U}\left(\hat{U}^{\prime} \hat{U} / T\right)^{-1} \hat{U}^{\prime}, \overline{\operatorname{SK}}\left(\nu_{0}\right)$ and $\overline{\mathrm{KU}}\left(\nu_{0}\right)$ are simulation-based estimates of the expected SK and KU given (2.8) and $\hat{p}\left[\operatorname{ESK}\left(\nu_{0}\right)\right]$ and $\hat{p}\left[\operatorname{EKU}\left(\nu_{0}\right)\right]$ are $p$-values, obtained by MC methods under (2.8). The MC test technique is also applied to obtain a size correct $p$-value for CSK. The CS for $\nu$ corresponds to the values of $\nu_{0}$ which are not rejected at level $\alpha_{1}$, using the latter $p$-value.

To conclude, we note that for the GARCH case, pre-estimating the $2 n \times 1$ vector $\phi$ is infeasible with 5 or even 10 year sub-samples of monthly data. Nevertheless, the single stage MMC is valid despite this limitation. Interestingly, the simulation study we report next suggests that power costs are unimportant even with relatively small samples.

\section{Simulation study}

We now present a small simulation study to assess the performance of the proposed methods. The design is calibrated to match our empirical analysis (see Section 8) which relies on monthly returns of 12 portfolios of NYSE firms over 1927-1995. We consider model (2.1) where $\tilde{R}_{\mathrm{M} t}$, $t=1, \ldots, T$, are the returns on the market portfolio from the aforementioned data over the last 5 and 10 year subperiods, as well as the whole sample. We thus take $n=12$ and $T=60,120$ and 828. The coefficients of (2.1) including $\gamma$ are set to their QML estimates (restricted under $\mathcal{H}_{\mathrm{B}}$ over the conformable sample period). From the QML regression, we also retain the estimated error covariance matrix, to generate model shocks; formally, we compute the corresponding empirical Cholesky factor (denoted $\hat{K}$ ) and use it for $K$ in (2.8). Test sizes with $K=I_{12}$ are also analyzed to illustrate the effects of portfolio repacking.

We consider normal and Student $t$-errors (with $\kappa=8$, in accordance with the kurtosis observed in the empirical application), so the random vectors $W_{t}, t=1, \ldots, T$, in (2.8) are generated following (2.9) and (2.10) respectively. The MC tests are applied imposing and ignoring information on $\kappa$, which allows us to document the cost of estimating this parameter. When $\kappa$ is considered unknown, MMC $p$-values are calculated over the interval $4 \leq \kappa \leq 13$ to keep execution time manageable (a wider range is allowed for the empirical application in Section 8). We also consider 
the case of GARCH errors (2.12), with $\phi_{1 i}=\phi_{1}$ and $\phi_{2 i}=\phi_{2}, i=1, \ldots, n$ (the coefficients are the same across equations). This restriction is motivated by execution time, but it is relaxed in Section 8. We use the diagonal elements of $\hat{K} \hat{K}^{\prime}$ to scale the intercept, yet we also consider the case where $\sigma_{i}^{2}=1, i=1, \ldots, n$. Samples are simulated with $\left(\phi_{1}, \phi_{2}\right)=(.15, .80)$. These parameters are treated, in turn, as known and as unknown quantities. In view of the low dimension of the nuisance parameter space in this case, when $\left(\phi_{1}, \phi_{2}\right)$ is treated as unknown, $p$-value maximization is achieved through a coarse grid search (for the purpose of this simulation). The $p$-value function does not appear to be very sensitive to the value of $\left(\phi_{1}, \phi_{2}\right)$, and the results presented below indicate this is sufficient for controlling test level in the relevant cases. A more thorough optimization is however used in Section 8.

The results of the simulation are summarized in Tables $1-3$. These tables report empirical rejection rates for various tests of $\mathcal{H}\left(\gamma_{0}\right)$ with nominal size $5 \%$. These rejection rates determine the coverage properties of confidence sets derived from the tests. Since we focus on estimating $\gamma, \mathcal{H}_{\mathrm{B}}$ is imposed for both the size and power studies. We compare the following tests: (1) a Wald-type test which rejects $\gamma=\gamma_{0}$ when $\gamma_{0}$ falls outside the Wald-type confidence interval $[\hat{\gamma}-$ $1.96 \times \operatorname{AsySE}(\hat{\gamma}), \hat{\gamma}+1.96 \times \operatorname{AsySE}(\hat{\gamma})]$, using the QML estimator $\hat{\gamma}$, an asymptotic standard error [AsySE $(\hat{\gamma})$, based on (2.24)], and a normal limiting distribution; (2) the MC and MMC tests based on the QLR test statistic $\operatorname{LR}\left(\gamma_{0}\right)$ defined in Theorem 3.1, with MC $p$-values for i.i.d. normal or Student- $t$ errors (with known or unknown $\kappa$ ), Gaussian GARCH with known or unknown $\left(\phi_{1}, \phi_{2}\right)$, as well as as $\phi_{1}=\phi_{2}=0$ (i.e., ignoring the GARCH dependence even when it is present in the simulated process); (3) tests based on the HAC Wald-type statistic $\mathcal{J}\left(\gamma_{0}\right)$ in (2.22), using a $\chi^{2}(n)$ critical value, $\mathrm{MC}$ with known $\left(\phi_{1}, \phi_{2}\right)$, and MMC where $\left(\phi_{1}, \phi_{2}\right)$ is taken as unknown.

In the size study (Table 1), $\gamma_{0}$ is calibrated to its QML counterpart from the data set $\left[\gamma_{0}=\right.$ -0.000089 for $T=60, \gamma_{0}=.004960$ for $T=120, \gamma_{0}=.005957$ for $\left.T=828\right]$. In the power study (tables 2 - 3), we focus on the $\hat{K}$ case; samples are drawn with $\gamma$ set to its QML estimate, and $\gamma_{0}$ is set to the latter value + step $\times \widehat{\sigma}_{i}^{\min }$, where $\widehat{\sigma}_{i}^{\min }=\left[\min \left\{\widehat{\sigma}_{i}^{2}\right\}\right]^{1 / 2}$, and $\widehat{\sigma}_{i}^{2}$ are the diagonal terms of $\hat{K} \hat{K}^{\prime}$ (with various step values). $N=99$ is used for MC tests $(N=999$ is used in the empirical application). In each experiment, the number of simulations is 1000 . We use 12 lags for the HAC correction.

Our results can be summarized as follows. The asymptotic i.i.d. or robust procedures are very unreliable from the viewpoint of controlling level. Whereas we observe empirical frequencies of type I errors over $70 \%$ and sometimes $90 \%$ with $T=60$, we still see empirical rejections near $55 \%$ with $T=828$. The results also show that the empirical size of the HAC-based tests is not affected by $K$, though a formal proof of its invariance is not available. This observation is however compatible with the fact that its size improves with larger samples: while the level of the Wald-type test shows no improvement (around 55\%) even with $T=828$ and normal errors, the size of the Wald-HAC statistic drops from $95 \%$ with $T=60$ to $12 \%$ with $T=828$. The LR and robust MC and MMC tests achieve level control; in the GARCH case, the MC LR test has the correct size even when GARCH dependence is not accounted for.

In view of the poor size performance of the asymptotic tests, the power study focuses on procedures whose level appears to be under control. Overall, the MMC correction is not too costly from the power viewpoint, with both Student- $t$ and GARCH errors. In the latter case, the LR-type test uncorrected for GARCH effects outperforms all the other tests. When GARCH corrections are performed via MMC, the LR-type test performs generally better than the Wald-HAC test. 
Table 1. Tests on zero-beta rate: empirical size

\begin{tabular}{|c|c|c|c|c|c|c|}
\hline$n=12$ & \multirow{2}{*}{\multicolumn{2}{|c|}{$\frac{T=60,}{K}$}} & \multicolumn{2}{|c|}{$T=120$} & \multicolumn{2}{|c|}{$T=828$} \\
\hline & & & & & & \\
\hline \multirow[t]{2}{*}{ Test } & $I_{12}$ & $\hat{K}$ & $I_{12}$ & $\hat{K}$ & $I_{12}$ & $\hat{K}$ \\
\hline & \multicolumn{6}{|c|}{ i.i.d. Normal } \\
\hline Wald-type & .709 & .196 & .633 & .096 & .578 & .050 \\
\hline \multirow[t]{2}{*}{$\operatorname{LR}\left(\gamma_{0}\right), \mathrm{MC}$} & .057 & .057 & .048 & .048 & .041 & .041 \\
\hline & \multicolumn{6}{|c|}{ i.i.d. Student- $t$} \\
\hline Wald-type & .714 & .218 & .645 & .106 & .587 & .055 \\
\hline$L R\left(\gamma_{0}\right), \mathrm{MC}, \kappa \mathrm{kno}$ & .053 & .053 & .046 & .046 & .043 & .043 \\
\hline \multirow[t]{2}{*}{$L R\left(\gamma_{0}\right)$, MMC, $\kappa$ unknown } & .043 & .043 & .035 & .035 & .031 & .031 \\
\hline & \multicolumn{6}{|c|}{ Gaussian GARCH } \\
\hline Wald-type & .676 & .200 & .628 & .086 & .579 & .047 \\
\hline$L R\left(\gamma_{0}\right), \mathrm{MC}, \phi_{1}=\phi_{2}=0$ & .059 & .059 & .048 & .048 & .046 & .046 \\
\hline $\mathcal{J}\left(\gamma_{0}\right), \chi^{2}(12)$ & .954 & .954 & .686 & .686 & .127 & .127 \\
\hline$J\left(\gamma_{0}\right)$, MMC, $\phi_{1}, \phi_{2}$ known & .049 & .049 & .045 & .045 & .049 & .040 \\
\hline$J\left(\gamma_{0}\right)$, MMC, $\phi_{1}, \phi_{2}$ unknown & .040 & .040 & .034 & .034 & .040 & .049 \\
\hline$L R\left(\gamma_{0}\right), \mathrm{MC}, \phi_{1}, \phi_{2}$ known & .064 & .064 & .043 & .043 & .050 & .028 \\
\hline$L R\left(\gamma_{0}\right), \mathrm{MMC}, \phi_{1}, \phi_{2}$ unknown & .054 & .054 & .032 & .032 & .028 & .050 \\
\hline
\end{tabular}

Note - The table reports the empirical rejection rates of various tests for $\mathcal{H}\left(\gamma_{0}\right)$ with nominal level $5 \%$. The values of $\gamma_{0}$ tested are: $\gamma_{0}=-0.000089$ for $T=60, \gamma_{0}=.004960$ for $T=120, \gamma_{0}=.005957$ for $T=828$. The design is calibrated to match our empirical analysis (see Section 8). The tests compared are the following. (1) A Wald-type test which rejects $\gamma=\gamma_{0}$ when $\gamma_{0}$ falls outside the Wald-type confidence interval $[\hat{\gamma}-1.96 \times \operatorname{AsySE}(\hat{\gamma}), \hat{\gamma}+1.96 \times$ $\operatorname{AsySE}(\hat{\gamma})]$, using the QML estimator $\hat{\gamma}$ with asymptotic standard error $[\operatorname{AsySE}(\hat{\gamma})]$ based on (2.24), and a normal limiting distribution. (2) MC and MMC tests based on $L R\left(\gamma_{0}\right)$ in (2.14), with MC $p$-values for i.i.d. normal and Student$t$ errors (with known or unknown $\kappa$ ), Gaussian GARCH with known or unknown $\left(\phi_{1}, \phi_{2}\right)$, as well as $\phi_{1}=\phi_{2}=0$ (i.e., ignoring the GARCH dependence even when it is present in the simulated process). (3) Tests based on the HAC Waldtype statistic $\mathcal{J}\left(\gamma_{0}\right)$ in (2.22), using a $\chi^{2}(n)$ critical value, MC with known $\left(\phi_{1}, \phi_{2}\right)$, and MMC where $\left(\phi_{1}, \phi_{2}\right)$ is taken as unknown. In the i.i.d. cases, the errors are generated using (2.8) with $K$ set to either $I_{12}$ or $\hat{K}$, which corresponds to the Cholesky factor of the least-squares error covariance estimate from the empirical data used for the simulation design. In the GARCH case, samples are generated with conditional variance as in (2.12) using $\hat{K}$ or $I_{12}$ for $K$. 
Table 2. Tests on zero-beta rate: empirical power Gaussian and Student designs

\begin{tabular}{ccccccc}
\hline$n=12$ & \multicolumn{2}{c}{$T=60$} & \multicolumn{2}{c}{$T=120$} & \multicolumn{2}{c}{$T=828$} \\
\hline Test & Step & Power & Step & Power & Step & Power \\
\hline$L R\left(\gamma_{0}\right)$, MC, $\phi_{1}=\phi_{2}=0$ & .50 & .151 & .50 & .226 & .20 & .129 \\
& .75 & .315 & .75 & .529 & .30 & .313 \\
& 1.0 & .544 & 1.0 & .835 & .50 & .814 \\
& 2.0 & .981 & 1.5 & .999 & .75 & .998 \\
\hline & & \multicolumn{5}{c}{ i.i.d. Student- $t$} \\
\hline$L R\left(\gamma_{0}\right)$, MC, $\kappa$ known & .50 & .134 & .50 & .181 & .20 & .109 \\
$L R\left(\gamma_{0}\right)$, MMC, $\kappa$ unknown & & .126 & & .158 & & .080 \\
$L R\left(\gamma_{0}\right)$, MC, $\kappa$ known & .75 & .264 & .75 & .428 & .30 & .237 \\
$L R\left(\gamma_{0}\right)$, MMC, $\kappa$ unknown & & .239 & & .384 & & .182 \\
$L R\left(\gamma_{0}\right)$, MC, $\kappa$ known & 1.0 & .494 & 1.0 & .709 & .50 & .660 \\
$L R\left(\gamma_{0}\right)$, MMC, $\kappa$ unknown & & .440 & & .673 & & .605 \\
$L R\left(\gamma_{0}\right)$, MC, $\kappa$ known & 2.0 & .939 & 1.5 & .997 & .75 & .966 \\
$L R\left(\gamma_{0}\right), M M C, \kappa$ unknown & & .925 & & .997 & & .960 \\
\hline
\end{tabular}

Note - The table reports the empirical rejection rates of various tests for $\mathcal{H}\left(\gamma_{0}\right)$ with nominal level $5 \%$. The values of $\gamma_{0}$ tested are: $\gamma_{0}=-0.000089$ for $T=60, \gamma_{0}=.004960$ for $T=120, \gamma_{0}=.005957$ for $T=828$. The sampling design conforms with the size study, for the $\hat{K}$ case. Samples are drawn with $\gamma$ calibrated to its QML counterpart from the 1991-95 subsample; values for $\gamma_{0}$ are set to the latter value + step $\times \widehat{\sigma}_{i}^{\min }$, where $\widehat{\sigma}_{i}^{\min }=\left[\min \left\{\widehat{\sigma}_{i}^{2}\right\}\right]^{1 / 2}$, and $\widehat{\sigma}_{i}^{2}$ are the diagonal terms of $\hat{K} \hat{K}^{\prime}$. See Table 1 for further details on the design and tests applied.

\section{Empirical analysis}

In this section, we assess $\mathcal{H}_{\mathrm{B}}$ as defined in (2.2) in the context of (2.1) under the distributional assumptions (2.10)-(2.11), as well as the Gaussian GARCH in (2.12). We use real monthly returns over the period going from January 1926 to December 1995, obtained from CRSP. The data studied involve 12 portfolios of NYSE firms grouped by standard two-digit industrial classification (SIC). The sectors studied include: (1) petroleum; (2) finance and real estate; (3) consumer durables; (4) basic industries; (5) food and tobacco; (6) construction; (7) capital goods; (8) transportation; (9) utilities; (10) textile and trade; (11) services; (12) leisure; for details on the SIC codes, see Beaulieu et al. (2007). For each month, the industry portfolios include the firms for which the return, the price per common share and the number of shares outstanding are recorded by CRSP. Furthermore, portfolios are value-weighted in each month. We measure the market return by value-weighted NYSE returns, and the real risk-free rate by the one month Treasury bill rate net of inflation, both available from CRSP. All MC tests were applied with $N=999$ and MMC $p$-values are obtained using the simulated annealing algorithm.

Our QML-based BCAPM test results are summarized in Table 4. Non-Gaussian $p$-values are the largest MC $p$-values over the error distribution parameters [respectively: $\kappa$ and $(\pi, \omega)$ for $(2.10)$ (2.11)] within the specified 97.5\% CSs; the latter are reported in Table 5. In the GARCH case (2.12), $p$-values are the largest MC $p$-values over all $\left(\phi_{1 i}, \phi_{2 i}\right)$. Given a $5 \%$ level test, the benchmark is 
Table 3. Tests on zero-beta rate: empirical power

Gaussian GARCH design

\begin{tabular}{|c|c|c|c|c|c|c|}
\hline$n=12$ & \multicolumn{2}{|c|}{$T=60$} & \multicolumn{2}{|c|}{$T=120$} & \multicolumn{2}{|c|}{$T=828$} \\
\hline Test & Step & Power & Step & Power & Step & Power \\
\hline$L R\left(\gamma_{0}\right), \mathrm{MC}, \phi_{1}=\phi_{2}=0$ & .50 & .112 & .50 & .203 & .20 & .195 \\
\hline $\mathcal{J}\left(\gamma_{0}\right)$, MMC, $\phi_{1}, \phi_{2}$ known & & .088 & & .155 & & .208 \\
\hline$J\left(\gamma_{0}\right)$, MMC, $\phi_{1}, \phi_{2}$ unknown & & .078 & & .133 & & .183 \\
\hline$L R\left(\gamma_{0}\right), \mathrm{MC}, \phi_{1}, \phi_{2}$ known & & .113 & & .204 & & .198 \\
\hline$L R\left(\gamma_{0}\right), \mathrm{MMC}, \phi_{1}, \phi_{2}$ unknown & & .106 & & .170 & & .168 \\
\hline$L R\left(\gamma_{0}\right), \mathrm{MC}, \phi_{1}=\phi_{2}=0$ & .75 & .247 & .75 & .449 & .30 & .465 \\
\hline$J\left(\gamma_{0}\right)$, MMC, $\phi_{1}, \phi_{2}$ known & & .177 & & .316 & & .442 \\
\hline$J\left(\gamma_{0}\right)$, MMC, $\phi_{1}, \phi_{2}$ unknown & & .158 & & .276 & & .411 \\
\hline$L R\left(\gamma_{0}\right), \mathrm{MC}, \phi_{1}, \phi_{2}$ known & & .248 & & .452 & & .471 \\
\hline$L R\left(\gamma_{0}\right), \mathrm{MMC}, \phi_{1}, \phi_{2}$ unknown & & .213 & & .411 & & .425 \\
\hline$L R\left(\gamma_{0}\right), \mathrm{MC}, \phi_{1}=\phi_{2}=0$ & 1.0 & .447 & 1.0 & .753 & .50 & .945 \\
\hline$J\left(\gamma_{0}\right), \mathrm{MC}, \phi_{1}, \phi_{2}$ known & & .300 & & .552 & & .934 \\
\hline$J\left(\gamma_{0}\right)$, MMC, $\phi_{1}, \phi_{2}$ unknown & & .269 & & .505 & & .920 \\
\hline$L R\left(\gamma_{0}\right), \mathrm{MC}, \phi_{1}, \phi_{2}$ known & & .441 & & .753 & & .950 \\
\hline$L R\left(\gamma_{0}\right), \mathrm{MMC}, \phi_{1}, \phi_{2}$ unknown & & .395 & & .709 & & .937 \\
\hline$L R\left(\gamma_{0}\right), \mathrm{MC}, \phi_{1}=\phi_{2}=0$ & 2.0 & .913 & 1.5 & .973 & .75 & 1.0 \\
\hline$J\left(\gamma_{0}\right)$, MMC, $\phi_{1}, \phi_{2}$ known & & .719 & & .856 & & 1.0 \\
\hline$J\left(\gamma_{0}\right)$, MMC, $\phi_{1}, \phi_{2}$ unknown & & .664 & & .931 & & 1.0 \\
\hline$L R\left(\gamma_{0}\right), \mathrm{MC}, \phi_{1}, \phi_{2}$ known & & .915 & & .970 & & 1.0 \\
\hline$L R\left(\gamma_{0}\right), \mathrm{MMC}, \phi_{1}, \phi_{2}$ unknown & & .892 & & .962 & & 1.0 \\
\hline
\end{tabular}

Note - The values of $\gamma_{0}$ tested are: $\gamma_{0}=-0.000089$ for $T=60, \gamma_{0}=.004960$ for $T=120, \gamma_{0}=.005957$ for $T=828$. Numbers reported are empirical rejection rates for various tests of $\mathcal{H}\left(\gamma_{0}\right)$ with nominal size $5 \%$. The sampling design conforms with the size study, for the $\hat{K}$ case; errors are generated with conditional variance as in (2.12) using $\hat{K}$. See Table 1 for a complete description of the designs and tests applied. Samples are drawn with $\gamma$ calibrated to its QML counterpart from the 1991-95 subsample; values for $\gamma_{0}$ are set to -the latter value + step $\times \widehat{\sigma}_{i}^{\text {min }}$ (for various step values) where $\widehat{\sigma}_{i}^{\min }=\left[\min \left\{\widehat{\sigma}_{i}^{2}\right\}\right]^{1 / 2}$, and $\widehat{\sigma}_{i}^{2}$ are the diagonal terms of $\hat{K} \hat{K}^{\prime}$.

.05 for $p_{\infty}$, normal LMC, MMC and GARCH $p$-values, while the Student- $t$ and normal mixture $p$-values should be compared to .025 (to ensure that the test has level .05). Non-rejections by LMC MC $p$-values are conclusive (though rejections are not); rejections based on the conservative bound reported under the heading BND are conclusive under normality; non-rejections based on the QML bound in the non-Gaussian case (reported under the heading BND) signal that the CS for $\gamma$ is not empty; however, since the MMC $p$-value is based on the tightest bound, this evidence does not necessarily imply non-rejection of $\mathcal{H}_{\mathrm{B}}$.

The empirical results presented in Table 4 show that the asymptotic test and the Gaussianbased bound test yield the same decision at level $5 \%$, although the former $p$-values are much lower. The non-normal $p$-values exceed the Gaussian-based $p$-value, enough to change the test decision. For instance, at the $5 \%$ significance level, we find seven rejections of the null hypothesis for the asymptotic $\chi^{2}(11)$ test, seven for the MC $p$-values under normality and with normal GARCH, and 
Table 4. QML-based tests of BCAPM

\begin{tabular}{|c|c|c|c|c|c|c|}
\hline \multirow[t]{2}{*}{ Sample } & \multirow[t]{2}{*}{$L R_{\mathrm{B}}$} & \multirow[t]{2}{*}{$p_{\infty}$} & \multicolumn{3}{|c|}{ Normal } & \multirow{2}{*}{$\frac{\text { GARCH }}{\text { BND }}$} \\
\hline & & & LMC & MMC & BND & \\
\hline $1927-30$ & 16.10 & .137 & .269 & .308 & .366 & .340 \\
\hline $1931-35$ & 14.09 & .228 & .344 & .381 & .432 & .451 \\
\hline $1936-40$ & 15.36 & .167 & .257 & .284 & .345 & .355 \\
\hline $1941-45$ & 18.62 & .068 & .148 & .163 & .203 & .213 \\
\hline $1946-50$ & 32.69 & .001 & .005 & .006 & .007 & .006 \\
\hline $1951-55$ & 37.04 & .000 & .003 & .004 & .004 & .003 \\
\hline $1956-60$ & 26.10 & .006 & .027 & .031 & .042 & .039 \\
\hline $1961-65$ & 29.21 & .002 & .011 & .016 & .020 & .015 \\
\hline $1966-70$ & 27.45 & .004 & .016 & .018 & .026 & .029 \\
\hline $1971-75$ & 16.81 & .113 & .213 & .224 & .292 & .294 \\
\hline $1976-80$ & 25.76 & .007 & .027 & .031 & .040 & .042 \\
\hline $1981-85$ & 14.98 & .183 & .316 & .335 & .387 & .404 \\
\hline $1986-90$ & 35.41 & .000 & .003 & .004 & .004 & .005 \\
\hline \multirow{3}{*}{$1991-95$} & 16.41 & .127 & .219 & .253 & .310 & .320 \\
\hline & \multicolumn{3}{|c|}{ Student- $t$} & \multicolumn{3}{|c|}{ Normal mixture } \\
\hline & LMC & MMC & $\mathrm{BND}$ & LMC & MMC & BND \\
\hline $1927-30$ & .272 & .316 & .360 & .279 & .313 & .381 \\
\hline $1931-35$ & .359 & .399 & .468 & .342 & .387 & .452 \\
\hline $1936-40$ & .282 & .308 & .372 & .265 & .302 & .357 \\
\hline $1941-45$ & .147 & .169 & .210 & .150 & .165 & .211 \\
\hline $1946-50$ & .007 & .007 & .010 & .007 & .007 & .008 \\
\hline $1951-55$ & .003 & .005 & .005 & .003 & .003 & .003 \\
\hline $1956-60$ & .030 & .040 & .052 & .028 & .035 & .045 \\
\hline $1961-65$ & .013 & .017 & .023 & .014 & .021 & .024 \\
\hline $1966-70$ & .020 & .025 & .032 & .018 & .023 & .028 \\
\hline $1971-75$ & .217 & .248 & .300 & .206 & .238 & .292 \\
\hline $1976-80$ & .026 & .035 & .039 & .026 & .034 & .042 \\
\hline $1981-85$ & .323 & .399 & .405 & .318 & .339 & .406 \\
\hline $1986-90$ & .004 & .005 & .005 & .004 & .004 & .005 \\
\hline $1991-95$ & .226 & .263 & .325 & .226 & .261 & .319 \\
\hline
\end{tabular}

Note $-L R_{\mathrm{B}}$ is the statistic in (2.18). Remaining numbers are associated $p$-values. $p_{\infty}$ is based on $\chi^{2}(n-1)$. All other non-Gaussian $p$-values are the largest MC $p$-values over the shape parameter $\nu$ within the specified CSs $[\nu=\kappa$ or $\nu=(\pi, \omega)$; refer to Table 5]. LMC is the bootstrap $p$-value in (6.14) and MMC is the maximal $p$-value in (6.13) (refer to Section 6.2). BND is the bound (6.2) for the Gaussian case and the $Q M L-B M C$ bound from Theorem 6.3 otherwise; the GARCH BND is the largest $Q M L-B M C$ over $\phi_{1 i}, \phi_{2 i}$ [from (2.12)]. Returns for the months of January and for October 1987 are excluded. Given a $5 \%$ level, the cut-off is .05 for $p_{\infty}$, the normal LMC, MMC and the GARCH $p$-values; for the Student- $\boldsymbol{t}$ and mixtures, the cut-off is .025 . $p$-values which lead to significant tests with this benchmark are in bold. 
Table 5. Confidence sets for intervening parameters

\begin{tabular}{c|ccccc|r}
\hline & \multicolumn{5}{|c}{ Mixture $(\pi, \omega)$, confidence set for $\omega$} & \multicolumn{1}{c}{$t(\kappa)$} \\
\cline { 2 - 7 } & $(1)$ & $(2)$ & $(3)$ & $(4)$ & $(5)$ & \multicolumn{1}{c}{$(6)$} \\
\cline { 2 - 7 } Sample & $\pi=0.1$ & $\pi=0.2$ & $\pi=0.3$ & $\pi=0.4$ & $\pi=0.5$ & \multicolumn{1}{c}{$\kappa$} \\
\hline $1927-30$ & $\geq 1.8$ & $1.6-2.8$ & $1.6-2.5$ & $1.6-2.5$ & $1.6-2.6$ & $3-12$ \\
$1931-35$ & $2.1-10.0$ & $1.9-3.0$ & $1.9-2.7$ & $1.9-2.7$ & $2.1-3.0$ & $3-8$ \\
$1966-40$ & $1.5-3.5$ & $1.5-2.3$ & $1.4-2.1$ & $1.4-2.0$ & $1.4-2.1$ & $4-25$ \\
$1941-45$ & $1.3-3.5$ & $1.3-2.1$ & $1.3-1.9$ & $1.3-1.8$ & $1.3-1.9$ & $\geq 5$ \\
$1946-50$ & $1.4-3.5$ & $1.3-2.2$ & $1.3-2.0$ & $1.3-1.9$ & $1.3-1.9$ & $5-37$ \\
$1951-55$ & $1.4-3.5$ & $1.4-2.2$ & $1.3-2.0$ & $1.3-1.9$ & $1.3-2.0$ & $5-34$ \\
$1956-60$ & $1.3-2.8$ & $1.2-2.0$ & $1.2-1.9$ & $1.2-1.8$ & $1.2-1.8$ & $\geq 5$ \\
$1961-65$ & $1.0-2.2$ & $1.0-1.6$ & $1.0-1.5$ & $1.0-1.5$ & $1.0-1.5$ & $\geq 7$ \\
$1966-70$ & $1.3-3.0$ & $1.3-2.0$ & $1.3-1.9$ & $1.3-1.8$ & $1.2-1.9$ & $\geq 5$ \\
$1971-75$ & $1.5-3.5$ & $1.5-2.2$ & $1.4-2.0$ & $1.4-1.9$ & $1.4-2.0$ & $4-24$ \\
$1976-80$ & $1.6-4.0$ & $1.5-2.5$ & $1.5-2.2$ & $1.5-2.2$ & $1.5-2.3$ & $4-19$ \\
$1981-85$ & $1.4-3.5$ & $1.4-2.1$ & $1.3-2.0$ & $1.3-1.9$ & $1.4-2.0$ & $5-33$ \\
$1986-90$ & $1.1-3.0$ & $1.1-2.0$ & $1.1-1.8$ & $1.0-1.7$ & $1.1-1.8$ & $\geq 5$ \\
$1991-95$ & $1.0-1.9$ & $1.0-1.5$ & $1.0-1.4$ & $1.0-1.3$ & $1.0-1.3$ & $\geq 19$ \\
\hline
\end{tabular}

Note - Numbers in columns (1)-(5) represent a CS for the parameters $(\pi, \omega)$ [respectively, the probability of mixing and the ratio of scales] of the multivariate mixtures-of-normal error distribution. Column (6) presents the CS for $\kappa$, the degrees-of-freedom parameter of the multivariate Student- $\boldsymbol{t}$ error distribution. See Section 6 for details on the construction of these CSs: the values of $(\pi, \omega)$ or $\kappa$ (respectively) in this set are not rejected by the $C S K$ test (6.15) [see Dufour et al. (2003)] under multivariate mixtures or Student- $t$ errors (respectively). Note that the maximum of the $p$-value occurs in the closed interval for $\omega$. Returns for the month of January and October 1987 are excluded from the data set.

five (relying on the MMC $p$-value) under the Student- $t$ and normal mixture distributions.

Focusing on Student $t$ and normal mixture distributions with parameters not rejected by proper GF tests, we see that mean-variance efficiency test results can change relative to the available $F$ based bound. The power advantages of the MMC procedure are illustrated by the results of the 1966-70 subperiod where the QML $p$-value exceeds $2.5 \%$ for the Student- $t$ and normal mixture distributions, whereas the MMC $p$-value signals a rejection.

The CSs for distributional parameters are reported in Table 5. In the mixture case, the confidence region is summarized as follows for presentation ease: we give the CS for $\omega$ corresponding to five different values of $\pi$.

In Table 6, we present: (i) the average real market return as well as the average real risk-free rate over each subperiod, (ii) the QML estimate of $\gamma$ (denoted $\hat{\gamma}$ ) and 95\% CSs for this parameter, using respectively the asymptotic standard errors (2.24) (under the heading Wald-type), and the LRtype tests with i.i.d. Gaussian, $t(\kappa)$ and normal mixture $(\pi, \omega)$ errors, plus Gaussian GARCH errors (lower panel). ${ }^{11}$ The values of $\gamma$ in the Fieller-type CS are not rejected by the test defined in Theorem

\footnotetext{
${ }^{11}$ Note that some values of $\hat{\gamma}$ are high. Nonetheless, comparing the average real market return for those subperiods with our estimate of $\gamma$ reveal that these high occurrences of $\gamma$ are consistent with subperiods during which the estimated zero-beta rate is higher than the market portfolio return. This is an illustration of finding, ex post, a linear relationship
} 
Table 6. QML-based point and set estimates for the zero-beta portfolio rate

\begin{tabular}{|c|c|c|c|c|}
\hline Sample & $\bar{R}_{M}$ & $\bar{r}_{f}$ & $\hat{\gamma}$ & Wald-type \\
\hline $1927-30$ & .0045 & .0045 & .0047 & {$[-.0037, .0130]$} \\
\hline $1931-35$ & .0103 & .0025 & -.0130 & {$[-.0301, .0039]$} \\
\hline $1926-40$ & .0031 & -.0006 & -.0069 & {$[-.0192, .0055]$} \\
\hline $1941-45$ & .0097 & -.0042 & .0117 & {$[.0037, .0198]$} \\
\hline $1946-50$ & .0021 & -.0051 & -.0219 & {$[-.0189,-.0070]$} \\
\hline $1951-55$ & .0145 & .0001 & .0024 & {$[-.0015, .0064]$} \\
\hline $1956-60$ & .0086 & .0002 & .0156 & {$[.0109, .0202]$} \\
\hline $1961-65$ & .0080 & .0014 & .0571 & {$[.0398, .0744]$} \\
\hline $1966-70$ & .0008 & .0004 & .0169 & {$[.0096, .0242]$} \\
\hline $1971-75$ & -.0061 & -.0010 & .0150 & {$[.0030, .0270]$} \\
\hline $1976-80$ & .0056 & -.0012 & -.0096 & {$[-.0169,-.0024]$} \\
\hline $1981-85$ & .0081 & .0037 & .0197 & {$[.0125, .0268]$} \\
\hline $1986-90$ & .0088 & .0020 & .0053 & {$[-.0024, .0131]$} \\
\hline \multirow[t]{2}{*}{$1991-95$} & .0104 & .0011 & .0010 & {$[-.0130, .0062]$} \\
\hline & \multicolumn{4}{|c|}{ 95\% Confidence set, Fieller-type } \\
\hline Sample & Normal errors & Student t errors & Mixture errors & GARCH \\
\hline $1927-30$ & {$[-.0133, .0227]$} & {$[-.0143, .0229]$} & {$[-.0141, .0227]$} & {$[-.0125, .020]$} \\
\hline $1931-35$ & {$[-.0509, .0225]$} & {$[-.0520, .0225]$} & {$[-.0157, .0227]$} & {$[-.0517, .0217]$} \\
\hline $1926-40$ & {$[-.0341, .0187]$} & {$[-.0350, .0190]$} & {$[-.0349, .0817]$} & {$[-.0300, .0175]$} \\
\hline $1941-45$ & {$[-.0045, .0275]$} & {$[-.0048, .0287]$} & {$[-.0045, .0283]$} & {$[-.0025, .0275]$} \\
\hline $1946-50$ & $\emptyset$ & $\emptyset$ & $\emptyset$ & $\emptyset$ \\
\hline $1951-55$ & $\emptyset$ & $\emptyset$ & $\emptyset$ & $\emptyset$ \\
\hline $1956-60$ & $\emptyset$ & {$[.0149, .0161]$} & $\emptyset$ & $\emptyset$ \\
\hline $1961-65$ & $\emptyset$ & $\emptyset$ & $\emptyset$ & $\emptyset$ \\
\hline $1966-70$ & $\emptyset$ & $\emptyset$ & $\emptyset$ & $\emptyset$ \\
\hline $1971-75$ & {$[-.0069, .0454]$} & {$[-.0081, .0488]$} & {$[-.0069, .0531]$} & {$[-.0050, .0450]$} \\
\hline $1976-80$ & $\emptyset$ & $\emptyset$ & $\emptyset$ & $\emptyset$ \\
\hline $1981-85$ & {$[.0059, .0371]$} & {$[.0051, .0376]$} & {$[.0051, .0387]$} & {$[.0075, .0350]$} \\
\hline $1986-90$ & $\emptyset$ & $\emptyset$ & $\emptyset$ & $\emptyset$ \\
\hline $1991-95$ & {$[-.0285, .0147]$} & {$[-.0303, .0154]$} & {$[-.0325, .0147]$} & {$[-.0275, .0125]$} \\
\hline
\end{tabular}

Note $-\bar{R}_{M}$ is the average real market portfolio return for each subperiod, $\bar{r}_{f}$ is the real average risk-free rate for each subperiod; $\hat{\gamma}$ is the QML estimate of $\gamma$; the remaining columns report $95 \%$ CSs for this parameter, using, respectively, the asymptotic standard errors (2.24) $[\hat{\gamma} \pm 1.96 \times \operatorname{AsySE}(\hat{\gamma})]$, the inverted test based on $L R\left(\gamma_{0}\right)$ from Theorem 3.1, and the MC Gaussian $p$-value, the MMC $p$-value imposing multivariate $t(\kappa)$ errors and mixture-of-normals $(\pi, \omega)$ errors, and the MMC GARCH $p$-value. See Section 4 for details on the construction of these CSs. Non-Gaussian $p$-values are the largest MC $p$-values over the shape parameters $\kappa$ or $(\pi, \omega)$. The GARCH $p$-value is the largest MC $p$-value over $\phi_{1 i}, \phi_{2 i}$ [from (2.12)]. Returns for the months of January and October 1987 are excluded from the data set. 
Table 7. Wald-HAC based inference on the zero-beta portfolio rate

\begin{tabular}{c|crrc}
\hline & $(1)$ & $(2)$ & $(3)$ & $(4)$ \\
\hline sample & $\tilde{\gamma}=\underset{\gamma_{0}}{\operatorname{argmin}} \mathcal{J}\left(\gamma_{0}\right)$ & $\min _{\gamma_{0}} \mathcal{J}\left(\gamma_{0}\right)$ & BND & $95 \%$ Confidence set, MMC \\
\hline $1927-30$ & .0090 & 71.29 & .650 & {$[-.0195, .0235]$} \\
$1931-35$ & -.0045 & 71.06 & .541 & {$[-.0240, .0250]$} \\
$1926-40$ & -.0045 & 54.52 & .620 & {$[-.0355, .0550]$} \\
$1941-45$ & .0415 & 163.26 & .143 & {$[-.0455, .0670]$} \\
$1946-50$ & .0000 & 133.76 & .121 & {$[-.0105, .0075]$} \\
$1951-55$ & .0075 & 104.93 & .250 & {$[.0000, .0120]$} \\
$1956-60$ & .0195 & 110.18 & .280 & {$[-.0385, .0415]$} \\
$1961-65$ & .0370 & 149.61 & .142 & {$[-.0295,-.0150] \cup[.0250, .0670]$} \\
$1966-70$ & .0090 & 168.54 & .081 & {$[.0045, .0135]$} \\
$1971-75$ & .0060 & 61.06 & .623 & {$[-.0180, .0067]$} \\
$1976-80$ & .0060 & 172.09 & .061 & {$[-.0225, .0135]$} \\
$1981-85$ & .0195 & 121.41 & .201 & {$[.0105, .0385]$} \\
$1986-90$ & .0030 & 184.38 & .030 & $\emptyset$ \\
$1991-95$ & .0100 & 53.60 & .841 & $\{\leq .0075\} \cup\{\geq .0310\}$ \\
\hline
\end{tabular}

Note $-\mathcal{J}\left(\gamma_{0}\right)$ is the HAC statistic in (2.22). $\tilde{\gamma}$ is the minimum distance estimator from (2.23). Column (3) provides a bound MC $p$-value simulated at $\tilde{\gamma}$ and maximized over $\phi_{1 i}, \phi_{2 i}$ [from (2.12)]. Column (4) provides the confidence set for $\gamma$ which inverts the inverted test based on $\mathcal{J}\left(\gamma_{0}\right)$ and the MMC GARCH $p$-value; again, this is the largest MC $p$-value over $\phi_{1 i}, \phi_{2 i}$ [from (2.12)]. Returns for the months of January and October 1987 are excluded from the data set. Given a $5 \%$ level, the cut-off the BND $p$-value is .05; $p$-values which lead to significant tests with this benchmark are in bold. Note that the CS which inverts $\mathcal{J}\left(\gamma_{0}\right)$ based on the asymptotic $\chi^{2}(12)$ cut-off is empty for all sub-periods.

3.1 to test $\mathcal{H}\left(\gamma_{0}\right)$. Rejection decisions are based on the largest MC $p$-values over all $\kappa$ and $(\pi, \omega)$ respectively; we did not restrict maximization to the CS for these parameters here. As expected in view of the $\mathcal{H}_{\mathrm{B}}$ test results, the exact CSs are empty for several subperiods. The usefulness of the asymptotic confidence intervals is obviously questionable here. Other results which deserve notice are the empty sets for 1956-60 subperiod; these sets correspond to the case where the efficiency bound test is significant (at $5 \%) .{ }^{12}$

To illustrate the differences between the asymptotic CS and ours, we next check whether the average real risk-free rate is contained in the CSs. For many subperiods, like 1966-70, the evidence produced by the asymptotic and MC Fieller-type confidence intervals is similar. There are nonetheless cases where the set estimates do not lead to the same decision. For instance, for 1941-45 and 1971-75, the average risk-free rate is not included in the asymptotic confidence interval, while it is covered by our MMC CSs. These are cases where, using the asymptotic confidence interval, the hypothesis $\gamma=r_{f}$ is rejected, whereas exact CSs indicate it should not be rejected. Conversely, in 1986-90, the asymptotic confidence interval includes the average risk-free rate, whereas our CSs are empty.

between risk and return with a negative slope. Furthermore, rerunning our analysis using 10-year subperiods leads to $\gamma$ estimates below the benchmark average return.

${ }^{12}$ This can be checked by referring to Table 4: although the reported maximal $p$-values in this table are performed over the confidence set for $\kappa$ and $(\pi, \omega)$, we have checked that the global maximal $p$-value leads to the same decision here. 
In Table 7, we report the Wald-HAC counterparts of the above QML-based tests (columns 2 and 3) as well as point and set estimates of $\gamma$ (columns 1 and 4). Column (2) reports the values of our proposed $J$-test-type minimum Wald-HAC statistic. In column (3), MMC refers to the maximal MC p-value [over all $\left(\phi_{1 i}, \phi_{2 i}\right)$ ] for this statistic, assuming the GARCH specification (2.12), and the level is $5 \%$; alternatively, an asymptotic $\chi^{2}(12)$ critical value (21.03 for a $5 \%$ level) can be used. In column (1), we report the GMM-type point estimate (denoted $\tilde{\gamma}$ ); the associated set estimate which inverts the Wald-HAC MC Gaussian GARCH based test is reported in column (4).

We first note that, on using the asymptotic critical value, a Wald-HAC test would reject the model in all subperiods at level 5\%. In contrast, the GARCH-MMC $p$-value is less than $5 \%$ only in the 1986-90 subperiod. In view of our simulation results from Section 7, these results illustrate the serious implications of asymptotic test size distortions. Recall that the LR-based MC and MMC (Gaussian and non-Gaussian, with and without GARCH) tests reject the model at the $5 \%$ level in at least three other sub-periods: 1946-50, 1950-55, 1960-65. This reflects the test relative power, as illustrated in Section 7. Turning to the estimates of $\gamma$, we note that the Wald-HAC based MMC CSs are substantially wider than the LR-based counterparts, only one CS is empty (in the 1986-1990 subperiods, in which case the model would be rejected), and the set is unbounded in the 1990-95 subperiod. Had we relied on the asymptotic $\chi^{2}(12)$ cut-off to invert the Wald-HAC test, all CSs would be empty. Again, these observations line up with our simulation results.

The above procedures applied to the full data yields empty CSs using the exact GARCH corrected LR and Wald-HAC criteria; the confidence interval using (2.24) is [.0007, .0088]. Since our subperiod analysis suggests that $\gamma$ is temporally unstable, one must be careful in interpreting such results. On using a Bonferroni argument (that accounts for time-varying parameters) based on the minimum (over subperiods) GARCH-corrected $p$-value which is .003 $<.05 / 12$, the model can be safely rejected at level $5 \%$, over the full sample.

\section{Conclusion}

This paper proposes exact mean-variance efficiency tests when the zero-beta (or risk-free) rate is not observable, which raises identification difficulties. Proposed methods are robust to this problem as well as to portfolio repacking, and allow for heavy-tailed return distributions. We also derive exact CSs for the zero-beta rate $\gamma$. While available Wald-type intervals are unreliable and lead to substantially different inference concerning $\gamma$, our CSs are valid in finite samples without assuming identification, and are empty by construction if efficiency is rejected.

We report a simulation study which illustrates the properties of our proposed procedures. Our results allow to disentangle "small-sample" problems from "asymptotic failures": whereas sample size, non-normality as well as parameter identification problems may concurrently cause finitesample distortions, identification issues are more pernicious and methods that assume identification away cannot be salvaged. We also examine efficiency of the market portfolio for monthly returns on NYSE CRSP portfolios. We find that efficiency is less rejected with non-normal assumptions. Exact CSs for $\gamma$ differ importantly from asymptotic ones, and LR-based CSs are tighter than their Wald counterparts. All CSs nevertheless suggest that $\gamma$ is not stable over time.

These results provide the motivation to extend our method to more general factor models, as discussed by Campbell et al. (1997, Chapter 6) and Shanken and Zhou (2007). These models raise the same statistical issues as the BCAPM, except that their definitional parameter is non-scalar. In this case, Fieller-type methods are clearly more challenging and raise worthy theoretical and 
empirical research questions.

\section{A. Appendix: Proofs}

Proof OF THEOREM 3.1 Under (2.8) and $\mathcal{H}\left(\gamma_{0}\right)$, we have: $T \hat{\Sigma}=\hat{U}^{\prime} \hat{U}=$ $K^{\prime} W^{\prime} M W K, \quad T \hat{\Sigma}\left(\gamma_{0}\right)=K^{\prime} W^{\prime} \bar{M}\left(\gamma_{0}\right) W K$. Then, under $\mathcal{H}\left(\gamma_{0}\right)$,

$$
\Lambda\left(\gamma_{0}\right)=\frac{\left|\hat{\Sigma}\left(\gamma_{0}\right)\right|}{|\hat{\Sigma}|}=\frac{\left|K^{\prime} W^{\prime} \bar{M}\left(\gamma_{0}\right) W K\right|}{\left|K^{\prime} W^{\prime} M(X) W K\right|}=\frac{\left|K^{\prime}\right|\left|W^{\prime} \bar{M}\left(\gamma_{0}\right) W\right||K|}{\left|K^{\prime}\right|\left|W^{\prime} M(X) W\right||K|}=\frac{\left|W^{\prime} \bar{M}\left(\gamma_{0}\right) W\right|}{\left|W^{\prime} M(X) W\right|}
$$

hence $\mathrm{P}\left[L R\left(\gamma_{0}\right) \geq x\right]=\mathrm{P}\left[T \ln \left(\left|W^{\prime} \bar{M}\left(\gamma_{0}\right) W\right| /\left|W^{\prime} M(X) W\right|\right) \geq x\right], \forall x$.

Proof of Lemma 5.1 The Gaussian log-likelihood function for model (2.5) is

$\ln [\tilde{L}(\tilde{Y}, C, \Sigma)]=-\frac{T}{2}[n(2 \pi)+\ln (|\Sigma|)]-\frac{1}{2} \operatorname{tr}\left[\Sigma^{-1}(\tilde{Y}-X C)^{\prime}(\tilde{Y}-X C)\right]=\ln [L(Y, B, \Sigma)]$.

Setting $\tilde{\Sigma}(C) \equiv \frac{1}{T}(\tilde{Y}-X C)^{\prime}(\tilde{Y}-X C)$, for any given value of $C, \ln [\tilde{L}(\tilde{Y}, C, \Sigma)]$ is maximized by taking $\Sigma=\tilde{\Sigma}(C)$ yielding the concentrated log-likelihood

$$
\ln \left[\tilde{L}(\tilde{Y}, C, \Sigma)_{c}=-\frac{n T}{2}[(2 \pi)+1]-\frac{T}{2} \ln (|\tilde{\Sigma}(C)|) .\right.
$$

The Gaussian MLE of $C$ thus minimizes $|\tilde{\Sigma}(C)|$ with respect to $C$. Let us denote by $\hat{C}(Y)$ the unrestricted MLE of $C$ so obtained, and by $\hat{C}\left(Y ; \gamma_{0}\right)$ and $\hat{C}_{\mathrm{B}}(Y)$ the restricted estimators subject to $\tilde{\mathcal{H}}\left(\gamma_{0}\right)$ and $\tilde{\mathcal{H}}_{\mathrm{B}}$ respectively. Suppose that $\tilde{Y}$ is replaced by $\tilde{Y}_{*}=\tilde{Y} A$ where $A$ is a nonsingular $n \times n$ matrix. We need to show that $L R_{*}\left(\gamma_{0}\right)=L R\left(\gamma_{0}\right)$ and $L R_{\mathrm{B} *}=L R_{\mathrm{B}}$, where $L R_{*}\left(\gamma_{0}\right)$ and $L R_{\mathrm{B} *}$ represent the corresponding test statistics based on the transformed data. Following this transformation, $|\tilde{\Sigma}(C)|$ becomes:

$$
\begin{aligned}
\left|\tilde{\Sigma}_{*}\left(C_{*}\right)\right| & =\left|\frac{1}{T}\left(\tilde{Y}_{*}-X C_{*}\right)^{\prime}\left(\tilde{Y}_{*}-X C_{*}\right)\right|=\left|\frac{1}{T} A^{\prime}\left(\tilde{Y}-X C_{*} A^{-1}\right)^{\prime}\left(\tilde{Y}-X C_{*} A^{-1}\right) A\right| \\
& =\left|A^{\prime} A\right|\left|\frac{1}{T}(\tilde{Y}-X C)^{\prime}(\tilde{Y}-X C)\right|=\left|A^{\prime} A\right||\tilde{\Sigma}(C)|
\end{aligned}
$$

where $C=C_{*} A^{-1}$. Then $\left|\tilde{\Sigma}\left(C_{*}\right)\right|$ is minimized by $\hat{C}_{*}\left(Y_{*}\right)=\hat{C}(Y) A$ and $\left|\tilde{\Sigma}_{*}\left(\hat{C}_{*}\left(Y_{*}\right)\right)\right|=$ $\left|A^{\prime} A\right| \mid \tilde{\Sigma}(\hat{C}(Y))$. On observing that $H\left(\gamma_{0}\right) C=0 \Longleftrightarrow H\left(\gamma_{0}\right) C A=0 \Longleftrightarrow H\left(\gamma_{0}\right) C_{*}=0$ for any $\gamma_{0}$, the restricted estimators of $C$ under $\tilde{\mathcal{H}}\left(\gamma_{0}\right)$ and $\tilde{\mathcal{H}}_{\mathrm{B}}$ are transformed in the same way: $\hat{C}_{*}\left(Y_{*} ; \gamma_{0}\right)=\hat{C}\left(Y ; \gamma_{0}\right) A$ and $\hat{C}_{* \mathrm{~B}}\left(Y_{*}\right)=\hat{C}_{\mathrm{B}}(Y) A$. This entails that $\left|\tilde{\Sigma}_{*}\left(\hat{C}_{*}\left(Y_{*} ; \gamma_{0}\right)\right)\right|=$ $\left|A^{\prime} A\right|\left|\tilde{\Sigma}\left(\hat{C}\left(Y ; \gamma_{0}\right)\right)\right|$ and $\left|\tilde{\Sigma}_{*}\left(\hat{C}_{* \mathrm{~B}}\left(Y_{*}\right)\right)\right|=\left|A^{\prime} A\right|\left|\tilde{\Sigma}\left(\hat{C}_{\mathrm{B}}(Y)\right)\right|$, so that

$$
\begin{aligned}
\tilde{\Lambda}_{*}\left(\gamma_{0}\right) & =\frac{\left|\tilde{\Sigma}_{*}\left(\hat{C}_{*}\left(Y_{*} ; \gamma_{0}\right)\right)\right|}{\left|\tilde{\Sigma}_{*}\left(\hat{C}_{*}\left(Y_{*}\right)\right)\right|}=\frac{\left|\tilde{\Sigma}\left(\hat{C}\left(Y ; \gamma_{0}\right)\right)\right|}{|\tilde{\Sigma}(\hat{C}(Y))|}=\tilde{\Lambda}\left(\gamma_{0}\right), \\
\tilde{\Lambda}_{\mathrm{B} *} & =\frac{\left|\tilde{\Sigma}_{*}\left(\hat{C}_{* \mathrm{~B}}\left(Y_{*}\right)\right)\right|}{\left|\tilde{\Sigma}_{*}\left(\hat{C}_{*}\left(Y_{*}\right)\right)\right|}=\frac{\left|\tilde{\Sigma}\left(\hat{C}_{\mathrm{B}}(Y)\right)\right|}{|\tilde{\Sigma}(\hat{C}(Y))|}=\tilde{\Lambda}_{\mathrm{B}} .
\end{aligned}
$$


Finally, in view of (2.14) and (2.20), we have $L R_{*}\left(\gamma_{0}\right)=T \ln \left[\tilde{\Lambda}_{*}\left(\gamma_{0}\right)\right]=T \ln \left[\tilde{\Lambda}\left(\gamma_{0}\right)\right]=$ $L R\left(\gamma_{0}\right)$ and $L R_{\mathrm{B} *}=T \ln \left(\tilde{\Lambda}_{\mathrm{B} *}\right)=T \ln (\tilde{\Lambda})=L R_{\mathrm{B}}$.

Proof of Theorem 5.2 Consider a transformation of the form $\tilde{Y}_{*}=\tilde{Y} K^{-1}$ or, equivalently, $Y_{*}=Y K^{-1}+\tilde{R}_{\mathrm{M}} \iota_{n}^{\prime}\left(I-K^{-1}\right)$. Using (2.1) and (2.8), we then have:

$$
\begin{aligned}
Y_{*} & =(X B+W K) K^{-1}+\tilde{R}_{\mathrm{M}} \iota_{n}^{\prime}\left(I-K^{-1}\right)=X B K^{-1}+\tilde{R}_{\mathrm{M}} \iota_{n}^{\prime}\left(I-K^{-1}\right)+W \\
& =\left(\iota_{T} a^{\prime}+\tilde{R}_{\mathrm{M}} \beta^{\prime}\right) K^{-1}+\tilde{R}_{\mathrm{M}} \iota_{n}^{\prime}\left(I-K^{-1}\right)+W \\
& =\tilde{R}_{\mathrm{M}} \iota_{n}^{\prime}+\left[\iota_{T} a^{\prime}+\tilde{R}_{\mathrm{M}}\left(\beta-\iota_{n}\right)^{\prime}\right] K^{-1}+W \\
& =\tilde{R}_{\mathrm{M}} \iota_{n}^{\prime}+X(B-\Delta) K^{-1}+W=\tilde{R}_{\mathrm{M}} \iota_{n}^{\prime}+X \bar{B}+W
\end{aligned}
$$

where $\bar{B}=(B-\Delta) K^{-1}$ and $\Delta=\left[0, \iota_{n}\right]^{\prime}$. Using Lemma 5.1, $L R\left(\gamma_{0}\right)$ and $L R_{\mathrm{B}}$ can be viewed as functions of $Y_{*}$, and depend on $(B, K)$ only through $\bar{B}=(B-\Delta) K^{-1}$. Under $\mathcal{H}_{\mathrm{B}}$, the nuisance parameter only involves $\gamma$ and $\left(\beta-\iota_{n}\right)^{\prime} K^{-1}$. Now the distribution of $L R\left(\gamma_{0}\right)$ and $L R_{\mathrm{B}}$ can be explicitly characterized by using (A.3) - (A.4) and observing that

$$
\begin{aligned}
\tilde{\Lambda}\left(\gamma_{0}\right) & =\frac{\left|\tilde{\Sigma}_{*}\left(\hat{C}_{*}\left(Y_{*} ; \gamma_{0}\right)\right)\right|}{\left|\tilde{\Sigma}_{*}\left(\hat{C}_{*}\left(Y_{*}\right)\right)\right|}=\frac{\left|\hat{W}\left(\gamma_{0}\right)^{\prime} \hat{W}\left(\gamma_{0}\right)\right|}{\left|\hat{W}^{\prime} \hat{W}\right|}, \\
\tilde{\Lambda}_{\mathrm{B}} & =\frac{\left|\tilde{\Sigma}_{*}\left(\hat{C}_{* \mathrm{~B}}\left(Y_{*}\right)\right)\right|}{\left|\tilde{\Sigma}_{*}\left(\hat{C}_{*}\left(Y_{*}\right)\right)\right|}=\frac{\inf \left\{\left|\tilde{\Sigma}_{*}\left(\hat{C}_{*}\left(Y_{*} ; \gamma_{0}\right)\right)\right|: \gamma_{0} \in \Gamma\right\}}{\left|\tilde{\Sigma}_{*}\left(\hat{C}_{*}\left(Y_{*}\right)\right)\right|}=\inf \left\{\tilde{\Lambda}\left(\gamma_{0}\right): \gamma_{0} \in \Gamma\right\},
\end{aligned}
$$

where $\hat{W}\left(\gamma_{0}\right)=\bar{M}\left(\gamma_{0}\right)\left(Y_{*}-\tilde{R}_{\mathrm{M}} \iota_{n}^{\prime}\right)=\bar{M}\left(\gamma_{0}\right)(X \bar{B}+W)=\bar{M}\left(\gamma_{0}\right)\left\{\left[\iota_{T} a^{\prime}+\tilde{R}_{\mathrm{M}}\left(\beta-\iota_{n}\right)^{\prime}\right] K^{-1}+\right.$ $W\}=\bar{M}\left(\gamma_{0}\right)\left\{\left[\iota_{T}\left(a^{\prime}+\gamma_{0}\left(\beta-\iota_{n}\right)^{\prime}\right)+\left(\tilde{R}_{\mathrm{M}}-\gamma_{0} \iota_{T}\right)\left(\beta-\iota_{n}\right)^{\prime}\right] K^{-1}+W\right\}=\bar{M}\left(\gamma_{0}\right)\left\{\iota_{T}(a+\right.$ $\left.\left.\gamma_{0}\left(\beta-\iota_{n}\right)^{\prime}\right) K^{-1}+W\right\}$ and $\hat{W}=M(X) W$. Under $\mathcal{H}_{\mathrm{B}}$ where $a=-\gamma\left(\beta-\iota_{n}\right), \hat{W}\left(\gamma_{0}\right)=$ $\left(\gamma_{0}-\gamma\right) \bar{M}\left(\gamma_{0}\right) \iota_{T}\left(\beta-\iota_{n}\right)^{\prime} K^{-1}+\bar{M}\left(\gamma_{0}\right) W$. The theorem then follows on observing that $L R\left(\gamma_{0}\right)=$ $T \ln \left[\tilde{\Lambda}\left(\gamma_{0}\right)\right]$ and $L R_{\mathrm{B}}=T \ln \left(\tilde{\Lambda}_{\mathrm{B}}\right)$. Further information can be drawn from the singular value decomposition of $\bar{B}$. Let $r$ be the rank of $\bar{B}$. Since $\bar{B}$ is a $2 \times n$ matrix, we have $0 \leq r \leq 2$ and we can write:

$$
\bar{B}=P D Q^{\prime}, \quad D=[\bar{D}, 0], \quad \bar{D}=\operatorname{diag}\left(\lambda_{1}^{1 / 2}, \lambda_{2}^{1 / 2}\right),
$$

where $D$ is a $2 \times n$ matrix, $\lambda_{1}$ and $\lambda_{2}$ are the two largest eigenvalues of $\bar{B}^{\prime} \bar{B}$ (where $\lambda_{1} \geq \lambda_{2} \geq 0$ ), $Q=\left[Q_{1}, Q_{2}\right]$ is an orthogonal $n \times n$ matrix whose columns are eigenvectors of $\bar{B}^{\prime} \bar{B}, Q_{1}$ is a $2 \times r$ matrix which contains eigenvectors associated with the non-zero eigenvalues of $\bar{B}^{\prime} \bar{B}, P=\left[P_{1}, P_{2}\right]$ is a $2 \times 2$ orthogonal matrix such that $P_{1}=\bar{B} Q_{1} D_{1}^{-1}$ and $D_{1}$ is a diagonal matrix which contains the non-zero eigenvalues of $\bar{B}^{\prime} \bar{B}$, setting $P=P_{1}$ and $D_{1}=\bar{D}$ if $r=2$, and $P=P_{2}$ if $r=0$; see Harville (1997, Theorem 21.12.1). Using Lemma 5.1 and Theorem 5.2, $L R\left(\gamma_{0}\right)$ and $L R_{\mathrm{B}}$ may then be reexpressed as:

$$
\begin{gathered}
L R\left(\gamma_{0}\right)=T \ln \left(\left|\tilde{W}\left(\gamma_{0}\right)^{\prime} \tilde{W}\left(\gamma_{0}\right)\right| /\left|\tilde{W}^{\prime} \tilde{W}\right|\right), \quad L R_{\mathrm{B}}=\inf \left\{L R\left(\gamma_{0}\right): \gamma_{0} \in \Gamma\right\} \\
\tilde{W}=\hat{W} Q=M(X) \bar{W}, \quad \bar{W}=W Q, \quad \tilde{W}\left(\gamma_{0}\right)=\hat{W}\left(\gamma_{0}\right) Q=\bar{M}\left(\gamma_{0}\right)(X P D+\bar{W}),
\end{gathered}
$$

$P D=[P \bar{D}, 0]$ and $P \bar{D}$ has at most 3 free coefficients ( $P$ is orthogonal). Under $\mathcal{H}_{\mathrm{B}}$,

$$
\tilde{W}\left(\gamma_{0}\right)=\bar{M}\left(\gamma_{0}\right) \iota_{T}\left[\left(\gamma_{0}-\gamma\right)\left(\varphi^{\prime} \varphi\right)^{1 / 2} \bar{\varphi}^{\prime}\right]+\bar{M}\left(\gamma_{0}\right) \bar{W}
$$




$$
\varphi=Q^{\prime}\left(K^{-1}\right)^{\prime}\left(\beta-\iota_{n}\right), \quad \bar{\varphi}=\varphi /\left(\varphi^{\prime} \varphi\right)^{1 / 2} .
$$

Define $\Phi=[\bar{\varphi}, \bar{\Phi}]$ as an orthogonal matrix such that $\Phi^{\prime} \Phi=\Phi \Phi^{\prime}=I_{n}$, so

$$
\Phi^{\prime} \Phi=\left[\begin{array}{cc}
\bar{\varphi}^{\prime} \bar{\varphi} & \bar{\varphi}^{\prime} \bar{\Phi} \\
\bar{\Phi}^{\prime} \bar{\varphi} & \bar{\Phi}^{\prime} \bar{\Phi}
\end{array}\right]=\left[\begin{array}{cc}
1 & 0 \\
0 & I_{n-1}
\end{array}\right], \quad \bar{\varphi}^{\prime} \Phi=\left[\begin{array}{cccc}
1 & 0 & \cdots & 0
\end{array}\right] .
$$

Then as in (A.7), $L R\left(\gamma_{0}\right)$ and $L R_{\mathrm{B}}$ may again be expressed under $\mathcal{H}_{\mathrm{B}}$ as:

$$
\begin{gathered}
L R\left(\gamma_{0}\right)=T \ln \left(\left|\tilde{W}_{B}\left(\gamma_{0}\right)^{\prime} \tilde{W}_{B}\left(\gamma_{0}\right)\right| /\left|\tilde{W}_{B}^{\prime} \tilde{W}_{B}\right|\right), \quad L R_{\mathrm{B}}=\inf \left\{L R\left(\gamma_{0}\right): \gamma_{0} \in \Gamma\right\} \\
\tilde{W}_{B}=\tilde{W} \bar{\Phi}=M(X) \bar{W}_{B}, \quad \bar{W}_{B}=\bar{W} \Phi \\
\tilde{W}_{B}\left(\gamma_{0}\right)=\tilde{W}\left(\gamma_{0}\right) \bar{\Phi}=\bar{M}\left(\gamma_{0}\right) \iota_{T} \varphi_{B}^{\prime}+\bar{M}\left(\gamma_{0}\right) \bar{W}_{B}
\end{gathered}
$$

where $\varphi_{B}^{\prime}=\left(\gamma_{0}-\gamma\right)\left(\varphi^{\prime} \varphi\right)^{1 / 2} \bar{\varphi}^{\prime} \Phi=\left(\gamma_{0}-\gamma\right)\left(\varphi^{\prime} \varphi\right)^{1 / 2}\left[\begin{array}{llll}1 & 0 & \cdots & 0\end{array}\right]$ which involves at most one free coefficient. When $W$ is non-Gaussian, the distributions of $L R\left(\gamma_{0}\right)$ and $L R_{\mathrm{B}}$ may be influenced by $\bar{B}$ through $Q$ in $\bar{W}$. Under the Gaussian assumption (2.9), the rows of $\bar{W}$ are i.i.d. $\mathrm{N}\left(0, I_{n}\right)$, so that $L R\left(\gamma_{0}\right)$ and $L R_{\mathrm{B}}$ follow distributions which depend on $(B, K)$ only through $P \bar{D}$. Under $\mathcal{H}_{\mathrm{B}}$, since the rows of $\bar{W}_{B}$ are i.i.d. $\mathrm{N}\left(0, I_{n}\right)$, this distribution involves only one nuisance parameter, in accordance with the result from Zhou (1991, Theorem 1), derived through a different method.

Proof of Theorem 6.1 $\mathcal{H}_{\mathrm{B}}=\cup_{\gamma_{0}} \mathcal{H}\left(\gamma_{0}\right)$. Since $L R_{\mathrm{B}}=\inf \left\{L R\left(\gamma_{0}\right): \gamma_{0} \in \Gamma\right\}$, we have $L R_{\mathrm{B}} \leq L R\left(\gamma_{0}\right)$, for any $\gamma_{0}$, hence $\mathrm{P}\left[L R_{\mathrm{B}} \geq x\right] \leq \mathrm{P}_{(B, K)}\left[L R\left(\gamma_{0}\right) \geq x\right], \forall x$, for each $\gamma_{0}$ and for any $(B, K)$ compatible with $\mathcal{H}\left(\gamma_{0}\right)$. Furthermore, under $\mathcal{H}_{\mathrm{B}}$, there is a value of $\gamma_{0}$ such that the distribution of $L R\left(\gamma_{0}\right)$ is given by Theorem 3.1, which entails (6.1). The result for the Gaussian special case then follows upon using (3.2).

Proof of Theorem 6.2 The result follows from (6.7), (3.6), and the inequalities $\hat{p}_{N}^{U}(\gamma, \nu) \leq$ $\hat{p}_{N}^{U}(\Gamma, \nu)$ and $\hat{p}_{N}^{U}(\gamma, \nu) \leq \hat{p}_{N}^{U}\left(\gamma, \Omega_{\mathcal{D}}\right) \leq \hat{p}_{N}^{U}\left(\Gamma, \Omega_{\mathcal{D}}\right)$.

Proof of TheOREM 6.3 When $\nu$ is specified, by (6.6), (2.19) and (3.5), we have: $\hat{p}_{N}^{U}(\hat{\gamma}, \nu) \equiv$ $p_{N}\left[L R_{\mathrm{B}}^{(0)} \mid \overline{L R}_{N}(\hat{\gamma}, \nu)\right]=p_{N}\left[L R^{(0)}(\hat{\gamma}) \mid \overline{L R}_{N}(\hat{\gamma}, \nu)\right]=\hat{p}_{N}(\hat{\gamma}, \nu)$, hence $\sup \left\{\hat{p}_{N}\left(\gamma_{0}, \nu\right): \gamma_{0} \in\right.$ $\Gamma\} \leq \alpha \Rightarrow \hat{p}_{N}(\hat{\gamma}, \nu) \leq \alpha \Rightarrow \hat{p}_{N}^{U}(\hat{\gamma}, \nu) \leq \alpha$; on noting that $\sup \left\{\hat{p}_{N}\left(\gamma_{0}, \nu\right): \gamma_{0} \in \Gamma\right\} \leq \alpha$ means that $C_{\gamma}(\alpha, \nu)$ is empty, $\hat{p}_{N}^{U}(\hat{\gamma}, \nu)>\alpha \Rightarrow \sup \left\{\hat{p}_{N}\left(\gamma_{0}, \nu\right): \gamma_{0} \in \Gamma\right\}>\alpha \Rightarrow C_{\gamma}(\alpha, \nu) \neq \emptyset$. For $\nu$ unknown,

$$
\begin{aligned}
\hat{p}_{N}^{U}\left(\hat{\gamma}, \Omega_{\mathcal{D}}\right) & =\sup \left\{\hat{p}_{N}^{U}\left(\hat{\gamma}, \nu_{0}\right): \nu_{0} \in \Omega_{\mathcal{D}}\right\}=\sup \left\{p_{N}\left[L R_{\mathrm{B}}^{(0)} \mid \overline{L R}_{N}\left(\hat{\gamma}, \nu_{0}\right)\right]: \nu_{0} \in \Omega_{\mathcal{D}}\right\} \\
& =\sup \left\{p_{N}\left[L R^{(0)}(\hat{\gamma}) \mid \overline{L R}_{N}\left(\hat{\gamma}, \nu_{0}\right)\right]: \nu_{0} \in \Omega_{\mathcal{D}}\right\}=\sup \left\{\hat{p}_{N}(\hat{\gamma}, \nu): \nu_{0} \in \Omega_{\mathcal{D}}\right\}
\end{aligned}
$$

hence $\sup \left\{\hat{p}_{N}\left(\gamma_{0}, \nu_{0}\right): \gamma_{0} \in \Gamma, \nu_{0} \in \Omega_{\mathcal{D}}\right\} \leq \alpha \Rightarrow \sup \left\{\hat{p}_{N}\left(\hat{\gamma}, \nu_{0}\right): \nu_{0} \in \Omega_{\mathcal{D}}\right\} \leq \alpha \Rightarrow$ $\hat{p}_{N}^{U}\left(\hat{\gamma}, \Omega_{\mathcal{D}}\right) \leq \alpha$ and $\hat{p}_{N}^{U}\left(\hat{\gamma}, \Omega_{\mathcal{D}}\right)>\alpha \Rightarrow \sup \left\{\hat{p}_{N}\left(\gamma_{0}, \nu_{0}\right): \gamma_{0} \in \Gamma, \nu_{0} \in \Omega_{\mathcal{D}}\right\}>\alpha \Rightarrow$ $C_{\gamma}(\alpha ; \mathcal{D}) \neq \emptyset$. 


\section{References}

Amsler, C. E. and Schmidt, P. (1985), 'A Monte Carlo investigation of the accuracy of multivariate CAPM tests', Journal of Financial Economics 14, 359-375.

Andrews, D. W. K., Moreira, M. J. and Stock, J. H. (2006), 'Optimal two-sided invariant similar tests for instrumental variables regression', Econometrica 74, 715-752.

Banz, R. W. (1981), 'Anomalies in relationships between securities' yields and yield surrogates', Journal of Financial Economics 6, 103-126.

Barone-Adesi, G., Gagliardini, P. and Urga, G. (2004), 'Testing asset pricing models with coskewness', Journal of Business and Economic Statistics 22, 474-485.

Bauwens, L., Laurent, S. and Rombouts, J. V. K. (2006), 'Multivariate GARCH models: a survey', Journal of Applied Econometrics 21, 79-109.

Beaulieu, M.-C., Dufour, J.-M. and Khalaf, L. (2005), Exact multivariate tests of asset pricing models with stable asymmetric distributions, in M. Breton and H. Ben Ameur, eds, 'Numerical Methods in Finance', Kluwer, Dordrecht, The Netherlands.

Beaulieu, M.-C., Dufour, J.-M. and Khalaf, L. (2007), 'Multivariate tests of mean-variance efficiency with possibly non-Gaussian errors: An exact simulation-based approach', Journal of Business and Economic Statistics 25, 398-410.

Beaulieu, M.-C., Dufour, J.-M. and Khalaf, L. (2009), 'Finite sample multivariate tests of asset pricing models with coskewness', Computational Statistics and Data Analysis 53, 2008-2021.

Black, F. (1972), 'Capital market equilibrium with restricted borrowing', Journal of Business 45, 444-454.

Black, F., Jensen, M. C. and Scholes, M. (1972), The capital asset pricing model: Some empirical tests, in M. C. Jensen, ed., 'Studies in the Theory of Capital Markets', Praeger.

Bolduc, D., Khalaf, L. and Yelou, C. (2008), 'Identification robust confidence sets methods for inference on parameter ratios with application to discrete choice models', Journal of Econometrics forthcoming.

Brennan, M. (1971), 'Capital market equilibrium with divergent borrowing and lending rates', Journal of Financial Economics 9, 3-18.

Campbell, J. Y., Lo, A. W. and MacKinlay, A. C. (1997), The Econometrics of Financial Markets, Princeton University Press, New Jersey.

Chen, N.-F., Roll, R. and Ross, S. A. (1986), 'Economic forces and the stock market', The Journal of Business 59, 383-403.

Chou, P.-H. (2000), 'Alternative tests of the zero-beta CAPM', The Journal of Financial Research 23, 469-493. 
Dufour, J.-M. (1989), 'Nonlinear hypotheses, inequality restrictions, and non-nested hypotheses: Exact simultaneous tests in linear regressions', Econometrica 57, 335-355.

Dufour, J.-M. (1997), 'Some impossibility theorems in econometrics, with applications to structural and dynamic models', Econometrica 65, 1365-1389.

Dufour, J.-M. (2003), 'Identification, weak instruments and statistical inference in econometrics', Canadian Journal of Economics 36(4), 767-808.

Dufour, J.-M. (2006), 'Monte Carlo tests with nuisance parameters: A general approach to finitesample inference and nonstandard asymptotics in econometrics', Journal of Econometrics 133, 443-478.

Dufour, J.-M. and Jasiak, J. (2001), 'Finite sample limited information inference methods for structural equations and models with generated regressors', International Economic Review 42, 815-843.

Dufour, J.-M. and Khalaf, L. (2002), 'Simulation based finite and large sample tests in multivariate regressions', Journal of Econometrics 111(2), 303-322.

Dufour, J.-M., Khalaf, L. and Beaulieu, M.-C. (2003), 'Exact skewness-kurtosis tests for multivariate normality and goodness-of-fit in multivariate regressions with application to asset pricing models', Oxford Bulletin of Economics and Statistics 65, 891-906.

Dufour, J.-M. and Taamouti, M. (2005), 'Projection-based statistical inference in linear structural models with possibly weak instruments', Econometrica 73, 1351-1365.

Dufour, J.-M. and Taamouti, M. (2007), 'Further results on projection-based inference in IV regressions with weak, collinear or missing instruments', Journal of Econometrics 139, 133-153.

Fama, E. F. (1965), ‘The behaviour of stock prices', Journal of Business 60, 401-424.

Fama, E. F. and French, K. R. (1993), 'Common risk factors in the returns on stocks and bonds', Journal of Financial Economics 33, 3-56.

Fama, E. F. and French, K. R. (2004), 'The capital asset pricing model: Theory and evidence', Journal of Economic Perspectives 18, 25-46.

Fama, E. F. and MacBeth, J. (1973), 'Risk, return, and equilibrium: Empirical tests', Journal of Political Economy 71, 607-636.

Fieller, E. C. (1954), 'Some problems in interval estimation', Journal of the Royal Statistical Society, Series B 16(2), 175-185.

Gibbons, M. R. (1982), 'Multivariate tests of financial models: A new approach', Journal of Financial Economics 10, 3-27.

Gibbons, M. R., Ross, S. A. and Shanken, J. (1989), 'A test of the efficiency of a given portfolio', Econometrica 57, 1121-1152. 
Harville, D. A. (1997), Matrix Algebra from a Statistician's Perspective, Springer-Verlag, New York.

Jensen, M. (1968), 'The performance of mutual funds in the period 1945-64', Journal of Finance 23, 389-416.

Jobson, J. D. and Korkie, B. (1982), 'Potential performance and tests of portfolio efficiency', Journal of Financial Economics 10, 433-466.

Kan, R., Robotti, C. and Shanken, J. (2008), Pricing model performance and the two-pass crosssectional regression methodology, Technical report, Rotman School of Management, University of Toronto, Toronto, Canada.

Kandel, S. (1984), 'The likelihood ratio test statistics of mean-variance efficiency without a riskless asset', Journal of Financial Economics 13, 575-592.

Kandel, S. (1986), 'The geometry of the likelihood estimator of the zero beta return', Journal of Finance 31, 339-346.

Kandel, S. and Stambaugh, R. F. (1989), 'A mean-variance framework for tests of asset pricing models', The Review of Financial Studies 2, 125-156.

Kim, D. (1995), 'The errors-in-variables problem in the cross-section of expected stock returns', Journal of Finance 50, 1605-1634.

Kleibergen, F. (2002), 'Pivotal statistics for testing structural parameters in instrumental variables regression', Econometrica 70, 1781-1803.

Kleibergen, F. (2005), 'Testing parameters in gmm without assuming that they are identified', Econometrica 73, 1103-1123.

Kleibergen, F. (2009), 'Tests of risk premia in linear factor models', Journal of Econometrics 149, 149-173.

Lewellen, J., Nagel, S. and Shanken, J. (2009), 'A skeptical appraisal of asset-pricing tests', Journal of Financial Economics forthcoming.

Litzenberger, G. N. and Ramaswamy, K. (1979), 'The effect of personal taxes and dividends on capital asset prices: Theory and empirical evidence', Journal of Financial Economics 7, 163-195.

MacKinlay, A. C. (1987), 'On multivariate tests of the Capital Asset Pricing Model', Journal of Financial Economics 18, 341-372.

MacKinlay, A. C. and Richardson, M. P. (1991), 'Using generalized method of moments to test mean-variance efficiency', The Journal of Finance 46, 511-527.

Mikusheva, A. (2009), Robust confidence sets in the presence of weak instruments, Technical report, MIT Department of Economics, Cambridge, Massachusetts.

Moreira, M. J. (2003), 'A conditional likelihood ratio test for structural models', Econometrica 71(4), 1027-1048. 
Perold, A. F. (2004), 'The capital asset pricing model', Journal of Economic Perspectives 18, 3-24.

Ravikumar, B., Ray, S. and Savin, E. (2000), 'Robust wald tests in sur systems with adding-up restrictions', Econometrica 68, 715-720.

Ray, S. and Savin, E. (2008), 'The performance of heteroskedasticity and autocorrelation robust tests: a Monte Carlo study with an application to the three-factor Fama-French asset-pricing model', Journal of Applied Econometrics 23, 91-109.

Richardson, M. and Smith, T. (1993), 'A test for multivariate normality in stock returns', Journal of Business 66, 295-321.

Roll, R. (1985), 'A note on the geometry of shanken's csr t2 test for mean/variance efficiency', Journal of Financial Economics 14, 349-357.

Sentana, E. (2009), 'The econometrics of mean-variance efficiency tests: A survey', Econometrics Journal 12, C65-C101.

Shanken, J. (1985), 'Multivariate tests of the zero-beta CAPM', Journal of Financial Economics 14, 325-348.

Shanken, J. (1986), 'Testing portfolio efficiency when the zero-beta rate is unknown: A note', Journal of Finance 41, 269-276.

Shanken, J. (1992), 'On the estimation of beta-pricing models', Review of Financial Studies 5, 1-33.

Shanken, J. (1996), Statistical methods in tests of portfolio efficiency: A synthesis, in G. S. Maddala and C. R. Rao, eds, 'Handbook of Statistics 14: Statistical Methods in Finance', NorthHolland, Amsterdam, pp. 693-711.

Shanken, J. and Zhou, G. (2007), 'Estimating and testing beta pricing models: Alternative methods and their performance in simulations', Journal of Financial Economics 84, 40-86.

Staiger, D. and Stock, J. H. (1997), 'Instrumental variables regression with weak instruments', Econometrica 65(3), 557-586.

Stewart, K. G. (1997), 'Exact testing in multivariate regression', Econometric Reviews 16, 321-352.

Stock, J. H. and Wright, J. H. (2000), 'GMM with weak identification', Econometrica 68, 10971126.

Stock, J. H., Wright, J. H. and Yogo, M. (2002), 'A survey of weak instruments and weak identification in generalized method of moments', Journal of Business and Economic Statistics 20(4), 518-529.

Velu, R. and Zhou, G. (1999), 'Testing multi-beta asset pricing models', Journal of Empirical Finance 6, 219-241.

Wang, J. and Zivot, E. (1998), 'Inference on structural parameters in instrumental variables regression with weak instruments', Econometrica 66(6), 1389-1404. 
Zerbe, G. O. (1978), 'On Fieller's theorem and the general linear model', The American Statistician 32(3), 103-105.

Zhou, G. (1991), 'Small sample tests of portfolio efficiency', Journal of Financial Economics 30, 165-191.

Zhou, G. (1995), 'Small sample rank tests with applications to asset pricing', Journal of Empirical Finance 2, 71-93.

Zivot, E., Startz, R. and Nelson, C. R. (1998), 'Valid confidence intervals and inference in the presence of weak instruments', International Economic Review 39, 1119-1144. 